(C) The Author(s), 2021. Published by Cambridge University Press on behalf of The Nutrition Society. This is an Open Access article, distributed under the terms of the Creative Commons Attribution licence (http://creativecommons.org/licenses/by/4.0/), which permits unrestricted re-use, distribution, and reproduction in any medium, provided the original work is properly cited.

\title{
Current perspectives on global sugar consumption: definitions, recommendations, population intakes, challenges and future direction
}

\author{
Janette Walton ${ }^{1}$ (1) , Haley Bell ${ }^{4}$, Roberta $\mathrm{Re}^{4}$ and Anne P Nugent ${ }^{2,3}$ (1) \\ ${ }^{1}$ Department of Biological Sciences, Munster Technological University, Cork, Republic of Ireland \\ ${ }^{2}$ Institute for Global Food Security, School of Biological Sciences, Queens University Belfast, Belfast, Northern Ireland \\ ${ }^{3}$ Institute of Food and Health, School of Agriculture and Food Sciences, University College Dublin, Belfield, Dublin 4, Ireland \\ ${ }^{4}$ Cambridge Food Science, Cambridge, UK
}

\begin{abstract}
Currently, there is considerable emphasis on the relationship between dietary sugar consumption and various health outcomes, with some countries and regions implementing national sugar reduction campaigns. This has resulted in significant efforts to quantify dietary sugar intakes, to agree on terms to describe dietary sugars and to establish associated recommendations. However, this information is infrequently collated on a global basis and in a regularised manner. The present review provides context regarding sugar definitions and recommendations. It provides a global review of the available data regarding dietary sugar intake, considering forms such as total, free and added sugars. A comprehensive breakdown of intakes is provided by age group, country and sugar form. This analysis shows that free sugar intakes as a percentage of total energy (\%E) are the highest for children and adolescents (12-14\%E) and the lowest for older adults (8\%E). This trend across lifecycle stages has also been observed for added sugars. The available data also suggest that, while some reductions in sugar intake are observed in a few individual studies, overall intakes of free/added sugars remain above recommendations. However, any wider conclusions are hampered by a lack of detailed high-quality data on sugar intake, especially in developing countries. Furthermore, there is a need for harmonisation of terms describing sugars (ideally driven by public health objectives) and for collaborative efforts to ensure that the most up-to-date food composition data are used to underpin recommendations and any estimates of intake or modelling scenarios.
\end{abstract}

Keywords: Free sugar: Added sugar: Dietary surveys: Dietary sugars: Sugar intake/consumption

(Received 26 July 2019; revised 6 July 2021; accepted 29 July 2021)

\section{Introduction}

Sugar has been described as a 'ubiquitous term', difficult to both define and measure, which may be in part due to its dual function as both a nutrient and as an ingredient ${ }^{(1)}$. A form of carbohydrate, chemically, the term 'sugars' usually describes mono- and disaccharides, with examples of monosaccharides being glucose, galactose and fructose and examples of disaccharides being sucrose, lactose and maltose. Examples of some natural contributing food sources include fruits (fructose, glucose, sucrose), honey (fructose, glucose, sucrose), milk and milk products (galactose, lactose), and table sugar (sucrose). Such '-ose' sugars can also form the building blocks of other carbohydrate types, such as oligosaccharides and polysaccharides. In addition, sugars in many of the above forms may be added as an ingredient to foods during processing or preparation to preserve food and/or to confer functional properties, such as viscosity, texture, body and colour, or at the table by consumers to add sweetness ${ }^{(1,2)}$. As a result, there is a broad range of foods containing sugars with varying quantities depending on the desired function, individual preference and food form. As a carbohydrate form, sugars, regardless of type, contribute $3.75 \mathrm{kcal} / \mathrm{g}$, which is equivalent to the contribution from starch.

Whilst the relationship between the frequency of consumption of foods containing sugars (particularly sucrose) and dental caries is well established ${ }^{(3-5)}$, there is debate concerning the relationship between dietary sugar intake and other health outcomes such as obesity, type 2 diabetes and cardiovascular disease. The evidence has been critiqued and systematically reviewed both in scientific publications ${ }^{(6,7)}$ and by advisory bodies and competent authorities ${ }^{(3-5,8,9)}$ with no firm conclusion that dietary sugars are directly related to diet-related diseases other than as a source of dietary energy particularly implicated as a contributor towards positive energy balance. As an individual dietary sugar, fructose has been identified of note, particularly with respect to metabolic health and hyperuricemia; however, as yet, no agreed conclusion has been reached regarding fructose ${ }^{(10,11)}$. The strongest available evidence of any link between dietary sugars and health is for body weight, and following the consumption of sugarsweetened beverages rather than sugars or a single sugar

* Corresponding author: Janette Walton, email janette.walton@mtu.ie 
Table 1. Definitions of sugars as used in dietary recommendations

\begin{tabular}{|c|c|c|}
\hline Term & Definition & Issuing body \\
\hline Total sugars & $\begin{array}{l}\text { Glycaemic carbohydrates composed of one to two monomers found in food. Includes monosaccharides } \\
\text { (glucose, fructose, galactose) and disaccharides (sucrose, lactose, maltose, trehalose) }\end{array}$ & $\begin{array}{l}\mathrm{FAO} / \mathrm{WHO}, 1998^{(22)} \\
\mathrm{EFSA}, 2010^{(3)}\end{array}$ \\
\hline Added sugars & $\begin{array}{l}\text { Sugars (mono- and disaccharides) used as ingredients in processed and prepared foods and sugars } \\
\text { eaten separately or added to foods at the table }\end{array}$ & $\begin{array}{l}\text { USDA/HHS, 2020(23) } \\
\text { IoM, 2005 (24) } \\
\text { EFSA } 2010^{(3)} \\
\text { Nordic Council of } \\
\quad \text { Ministers, } 2012^{(25)}\end{array}$ \\
\hline Free sugars & $\begin{array}{l}\text { Includes monosaccharides (glucose, fructose and galactose) and disaccharides (sucrose, lactose, } \\
\text { maltose and trehalose) added to foods by the manufacturer, cook, or consumer plus sugars naturally } \\
\text { present in honey, syrups, fruit juices and fruit juice concentrates }\end{array}$ & $\begin{array}{l}\text { WHO 2003, } 2015^{(4,26)} \\
\text { SACN } 2015^{(5)}\end{array}$ \\
\hline $\begin{array}{l}\text { Non-milk extrinsic } \\
\text { sugars }\end{array}$ & $\begin{array}{l}\text { Sugars not located within the cellular structure of a food, such as those found in fruit juice, honey and } \\
\text { syrups, and those added to processed foods, excluding lactose in milk. Definition originated in UK } \\
\text { and in } 2015 \text { use was superseded by use of 'free sugars' }\end{array}$ & UK DoH $1991^{(27)}$ \\
\hline
\end{tabular}

FAO/WHO (Food and Agriculture Organization and the World Health Organization), 1998. Carbohydrates in human nutrition. Report of a Joint FAO/WHO Expert Consultation, Rome, Italy, 14-18 April 1997. EFSA NDA Panel (EFSA Panel on Dietetic Products, Nutrition and Allergies), 2010. Scientific Opinion on Dietary Reference Values for carbohydrates and dietary fibre.

EFSA Journal 2010;8(3): 1462, 77 pp. https://doi.org/10.2903/j.efsa.2010.1462.

IoM (Institute of Medicine), 2002. Dietary reference intakes for energy, carbohydrate, fiber, fat, fatty acids, cholesterol, protein, and amino acids.

per $s e^{(5,12,13)}$. At the request of a number of countries in the wider Nordic region, the European Food Safety Authority (EFSA) is currently (2020) undertaking a renewed review of the evidence to provide scientific advice on dietary sugars and to derive a tolerable upper intake level (UL) for total/added/free dietary sugars ${ }^{(14)}$. A draft for public consultation is expected in mid-2021.

In light of this continuing focus on sugars and their role in health, it is important to review current estimates of intake globally and to place these in context against recommendations. This allows generation of a benchmark for ongoing surveillance. Previously, there have been three reviews covering dietary sugars intakes, one with a global perspective ${ }^{(15)}$ a second focused on European countries $^{(16)}$ and a third focusing on Latin American countries ${ }^{(17)}$. However, there exists a need to capture more recent data on a global basis and in a consistent balanced manner. The current review provides an up-to date critique of sugars in terms of their classification and dietary recommendations. A secondary analysis of the available data on dietary sugar intakes worldwide is described, followed by a perspective on the role of sugars in the diet globally.

\section{Classification and definitions of dietary sugars}

The term 'sugars' is conventionally used to define the mono- and disaccharides in foods (also known as total sugars). This includes naturally occurring sugars as well as those added to a food or drink (such as sucrose, fructose, maltose, lactose, honey, syrup, corn syrup, high-fructose corn syrup, molasses and fruit juice concentrate) but does not include polyols. Other definitions of dietary sugars are typically either linked to function in terms of establishing dietary recommendations, or they inform regulations and food labelling. With respect to definitions used to underpin dietary recommendations, the key terms are 'total sugars' (as defined above), 'free sugars', 'added sugars' and, historically, 'non-milk extrinsic sugars' (UK only). Table 1 provides a brief description of these definitions and issuing guidance body.
Of note, the term 'total sugars' provides the most encompassing measure of dietary sugars intake, used widely in food labelling and for informing consumers about the total sugars content of foods and drinks. The use of the term 'total sugars' in food labelling is mainly due to there currently being no accepted method for empirically measuring free sugars or non-milk extrinsic sugars in a food/drink. However, it is less frequently used in dietary recommendations ${ }^{(18)}$. Terms that are used more frequently in dietary recommendations include 'free' or 'added' sugars ${ }^{(19)}$. Both EFSA (Europe) and the Institute of Medicine (United States) define added sugars as all sugars that are added to foods and beverages by the manufacturer, cook and consumer, but do not include natural sources of sugars such as those included in unsweetened fruit juices ${ }^{(3,20)}$. On the other hand, free sugars, as defined by WHO and EFSA, include all added sugars but also those sugars naturally present in honey, syrups and fruit juices ${ }^{(4,21)}$. Such divergence in definitions for sugars is reflected in varying descriptions when reporting on dietary intake, in varying use of the terms in food regulations and even in defining the composition of formulae and foods for infants and young children ${ }^{(1)}$.

\section{Dietary guidelines for sugars consumption}

Universally, most food-based dietary guidelines make the sensible and pragmatic suggestion for populations to limit the consumption of foods of low nutrient density, such as foods of low dietary quality that are high in calories, sugar, salt and/or fat, especially saturated fat. In recent years, many government and health organisations worldwide have revisited their guidance for dietary sugar intake, particularly in relation to maximum recommended intakes for added or free sugars. Some of these goals are average population maximum targets, and some are recommendations for intakes at the individual level. Examples of current recommendations relating to quantitative guidelines for dietary sugars are included in Table 2 and are summarised briefly below. 
For free sugars, the World Health Organisation (WHO) recommends that all individuals, both adults and children, reduce their intake of free sugars to $<10 \%$ energy (E) intake and suggest a further conditional recommendation of reduction to $<5 \% \mathrm{E}$ for further health benefits ${ }^{(4)}$. This $<5 \% \mathrm{E}$ value is also proposed by both the UK Scientific Advisory Committee on Nutrition (SACN) (as an average population maximum target) and by the European Society for Paediatric Gastroenterology Hepatology and Nutrition (ESPGHAN) (as individual guidance). Of note, there is a differing body of evidence underpinning these recommendations. Whilst SACN focus on intervention and prospective cohort studies relating to the intake of sugars to energy intake and to an increased risk of dental caries and type 2 diabetes mellitus ${ }^{(5)}$, the ESPGHAN recommendation ${ }^{(1)}$ is informed by the conclusions of both $\mathrm{WHO}$ and $\mathrm{SACN}^{(4,5)}$ rather than by independent analyses. Of note also, ESPGHAN suggest children under 2 years of age should have even lower intakes than the $<5 \% \mathrm{E}^{(1)}$.

For added sugars, both the Nordic countries and the United States recommend limiting the intake of added sugars to $<10 \%$ $\mathrm{E}^{(23,25)}$. In both cases, these recommendations were in the context of healthy dietary patterns rather than any link with disease risk, a major difference to recommendations for free sugars. Whilst the American Heart Association (AHA) in the United States have listed a UL for children ${ }^{(29)}$, this was not reflected in US national guidelines, which were unable to establish this guidance value owing to lack of evidence. In the absence of such scientific evidence, the IoM suggested that intakes above $25 \% \mathrm{E}$ from added sugars may be associated with displacement of foods that are major sources of essential micronutrients, but they were unable to set a UL at that time (2002) and have not set one since ${ }^{(9)}$. Of note, the French Agency for Food, Environmental and Occupational Health \& Safety (ANSES) have set an upper limit for total sugars of $100 \mathrm{~g} / \mathrm{d}$ for adults, but this excludes lactose and galactose naturally present in milk and dairy products ${ }^{(8)}$. As mentioned previously, the EFSA is currently mandated to provide scientific advice on dietary sugars and to establish a UL if the available data allow it, having previously being unable to set such a benchmark in $2010^{(3)}$. It is unknown yet how an establishment of such a UL may influence national dietary guidelines within Europe: currently, whilst some European countries have quantitative limits established, such as the Nordic countries, the UK and France, others such as Germany have adopted the WHO recommendations ${ }^{(28)}$. What is currently known regarding the EFSA scientific evaluation is that the exposure assessment will focus on intake of free sugars and that the disease endpoints of interest will include incidence/severity of dental caries and incidence of metabolic diseases. Other less direct endpoints will include measures of body fatness, ectopic fat distribution, glucose homeostasis and blood lipids among others ${ }^{(14)}$. As we await the findings of this EFSA scientific opinion, recent reviews suggest that, for numerical dietary sugar recommendations, more explicit quantification, justifications and communication of uncertainty factors, together with greater international harmonisation for both exposures and outcomes, would help to minimise any variation in recommendations ${ }^{(17,19,30)}$. An update of these reviews is included below.

\section{Sugar intake data}

\section{Selection of data}

Recent reviews of dietary surveys (published between 2014 and 2018) were used to identify a list of countries that have undertaken dietary surveys and the dates that these surveys were performed ${ }^{(31-34)}$. These were then supplemented with searches of databases such as Science Direct, PubMed and Google Scholar using terms such as 'national dietary survey' and 'sugar(s) consumption'. As nutrient and food intakes derived from national food consumption survey data are typically published in reports, rather than peer-reviewed papers, the data presented in the present review were primarily collected from various reports and publications of national dietary surveys. Surveys and reports known to the authors of the present study were also included. Peer review publications were used to obtain additional data, for countries including New Zealand, Mexico, Spain, Brazil, the United States and Ireland. Studies were included if the data were (i) nationally representative, (ii) available in the public domain, (iii) summarised (no raw data were analysed) and (iv) collected at the individual level, and if (v) sugar intake was reported as a nutrient rather than a food group. There was no limit to the study size, and data from all available direct dietary assessment methodology were included, including food diaries, diet recalls or food frequency questionnaires.

\section{Presentation of data}

In an attempt to assess the available evidence which quantifies dietary sugar intake, a secondary analysis of published intake data for all sugar forms has been completed, and the findings (as reported in their original form) are reported in Tables 3-7 and summarised below. Energy values have been included to allow others to make crude estimates of sugars intakes as a percentage of energy where these data were not in the original reports.

Values are reported for estimated intakes of energy, total sugars, added sugars, free sugars or sucrose as available. Where energy was provided in kcal, it was converted to MJ using a factor of $238 \cdot 85$. Data are presented as provided in the reports and publications, and where possible, data have been divided by age range and not subdivided by sex. No statistical analysis has been performed on these data sets, and the results are purely descriptive. Under-reporting of food consumption can influence the accuracy of dietary intake data, resulting in an underestimate of energy and nutrients. The data presented in the tables do not account for under-reporting as under-reporters are not typically excluded from data presented in reports of food and nutrient consumption. Additionally, the data are usually presented as a percentage of energy intake, which partially accounts for this but will still result in an underestimate of sugar intake if foods/drinks that are high in sugar are selectively underreported. It should also be noted that there are significant limitations in comparing sugar intakes across countries (from different time points) since the composition of individual foods and beverages may have changed drastically over that time and may not be reflective of current consumption. 
Table 2. Examples of dietary reference values for sugars globally

\begin{tabular}{|c|c|c|c|c|c|}
\hline Organisation & Population group & Year & Recommendation & Evidence base & Reference \\
\hline German Nutrition Society & $\begin{array}{l}\text { Children and } \\
\text { adults }\end{array}$ & 2019 & $\begin{array}{l}\text { Maximum free sugar intake of no more than } 10 \% \text { of total } \\
\text { energy intake }\end{array}$ & Adopted from $\mathrm{WHO}$ & Ernst et al. $2019^{(28)}$ \\
\hline $\begin{array}{l}\text { European Society for Paediatric } \\
\text { Gastroenterology, Hepatology } \\
\text { and Nutrition (ESPGHAN) } \\
\text { Committee on Nutrition }\end{array}$ & Children & 2017 & $\begin{array}{l}\text { Intakes of free sugars should be reduced in children and } \\
\text { adolescents }(2-18 \text { years) with a desirable upper limit } \\
\text { of less than } 5 \% \text { energy intake and should be even } \\
\text { lower in those younger than } 2 \text { years }\end{array}$ & $\begin{array}{l}\text { Built on the conclusions of other authoritative bodies } \\
\text { (WHO, 2015, SACN, 2015, Vos, 2016) }\end{array}$ & Fidler et al. $2017^{(1)}$ \\
\hline $\begin{array}{l}\text { American Heart Association } \\
(\mathrm{AHA})\end{array}$ & Children & 2017 & $\begin{array}{l}\text { Children should consume } \leq 25 \mathrm{~g} \text { (100 kcal or } \approx 6 \text { tea- } \\
\text { spoons) of added sugars per day, and added sugars } \\
\text { should be avoided for children }<2 \text { years of age }\end{array}$ & & Vos et al. $2017^{(29)}$ \\
\hline $\begin{array}{l}\text { French Agency for Food, } \\
\text { Environmental and } \\
\text { Occupational Health \& Safety } \\
\text { (ANSES) }\end{array}$ & Adult population & 2016 & $\begin{array}{l}\text { Upper intake limit for total sugars of } 100 \mathrm{~g} / \mathrm{d} \text { (excluding } \\
\text { lactose and galactose naturally present in milk and } \\
\text { dairy products) }\end{array}$ & $\begin{array}{l}\text { Based on calculation from minimum daily intake of fruc- } \\
\text { tose for which a significant increase in blood triacylgly- } \\
\text { cerol were observed (in intervention studies) }\end{array}$ & ANSES, $2016^{(8)}$ \\
\hline $\begin{array}{l}\text { World Health Organisation } \\
\text { (WHO) }\end{array}$ & $\begin{array}{l}\text { Children and } \\
\text { adults }\end{array}$ & 2015 & $\begin{array}{l}\text { Intake of free sugars should not exceed } 10 \% \text { of total } \\
\text { energy intake (strong recommendation) and should be } \\
\text { less than } 5 \% \text { of total energy intake (conditional rec- } \\
\text { ommendation) }\end{array}$ & $\begin{array}{l}\text { Based on risk of non-communicable diseases with a par- } \\
\text { ticular focus on weight gain and dental caries }\end{array}$ & $\mathrm{WHO}, 2015^{(4)}$ \\
\hline $\begin{array}{l}\text { U.S. Department of Health and } \\
\text { Human Services and U.S. } \\
\text { Department of Agriculture } \\
\text { (2015) }\end{array}$ & $\begin{array}{l}\text { General } \\
\text { Population }\end{array}$ & 2020 & $\begin{array}{l}\text { Consume less than } 10 \% \text { of calories per day from added } \\
\text { sugars }\end{array}$ & $\begin{array}{l}\text { Target based on food pattern modelling and national data } \\
\text { on intakes of calories from added sugars that demon- } \\
\text { strate the public health need to limit calories from } \\
\text { added sugars to meet food group and nutrient needs } \\
\text { within calorie limits }\end{array}$ & USDA, 2020(23) \\
\hline $\begin{array}{l}\text { Scientific Advisory Committee } \\
\text { on Nutrition (SACN) UK }\end{array}$ & 2 years and over & 2015 & $\begin{array}{l}\text { Recommended that the average population intake of free } \\
\text { sugars should not exceed } 5 \% \text { of total energy intake } \\
\text { for age groups from } 2 \text { years upwards }\end{array}$ & $\begin{array}{l}\text { Based on evidence of increased energy from associations } \\
\text { with an increased risk of dental caries. Additional evi- } \\
\text { dence related to that of sugar-sweetened beverages } \\
\text { and weight gain in children and adolescents and an } \\
\text { increased risk of type } 2 \text { diabetes in adults }\end{array}$ & SACN, $2015^{(5)}$ \\
\hline $\begin{array}{l}\text { Nordic Nutrition } \\
\quad \text { Recommendations (NNR) }\end{array}$ & $\begin{array}{l}\text { General Nordic } \\
\text { population }\end{array}$ & 2012 & $\begin{array}{l}\text { Limit the intake of added sugars (sucrose, fructose, and } \\
\text { starch hydrolysates) to }<10 \% \text { of the total energy } \\
\text { intake }\end{array}$ & $\begin{array}{l}\text { Based on ensuring adequate intakes of dietary fibre and } \\
\text { micronutrients }\end{array}$ & NNR, 2012 (25) \\
\hline $\begin{array}{l}\text { European Food Safety Authority } \\
\text { (EFSA) }\end{array}$ & $\begin{array}{l}\text { General popula- } \\
\text { tion }\end{array}$ & 2010 & $\begin{array}{l}\text { Insufficient data to set an upper limit for (added) sugars } \\
\text { intake. }\end{array}$ & & EFSA, $2010^{(3)}$ \\
\hline US Institute Of Medicine (IoM) & $\begin{array}{l}\text { General popula- } \\
\text { tion }\end{array}$ & 2005 & Maximum intake level of $\leq 25 \%$ total energy & $\begin{array}{l}\text { Based on prevention of displacement of foods that are a } \\
\text { major source of micronutrients }\end{array}$ & IoM, 2005(20) \\
\hline
\end{tabular}


Table 3. Energy and sugar intake of infants and young children $(<4$ years)

\begin{tabular}{|c|c|c|c|c|c|c|c|c|c|c|c|c|c|c|c|c|c|c|c|c|c|c|}
\hline \multirow[b]{2}{*}{ Country } & \multirow[b]{2}{*}{ Assessment year } & \multirow[b]{2}{*}{ Age range (years) } & \multirow[b]{2}{*}{$n$} & \multirow[b]{2}{*}{ Method } & \multirow[b]{2}{*}{ MJ } & \multirow{2}{*}{$\frac{\text { Energy }}{\mathrm{SD}}$} & \multicolumn{4}{|c|}{ Total sugars } & \multicolumn{4}{|c|}{ Added sugars } & \multicolumn{4}{|c|}{ Sucrose } & \multicolumn{4}{|c|}{ Free sugars } \\
\hline & & & & & & & g & SD & $\% \mathrm{E}$ & SD & g & SD & $\% \mathrm{E}$ & SD & g & SD & $\% \mathrm{E}$ & SD & g & SD & $\% \mathrm{E}$ & SD \\
\hline \multirow[t]{2}{*}{ Australia ${ }^{(35,36)}$} & 2011-2012 & 2-3 (M) & & 24-h recall & $6 \cdot 0$ & & $94 \cdot 3$ & & 24.9 & & 32.5 & & $8 \cdot 4$ & & & & & & $42 \cdot 2$ & & 11.0 & \\
\hline & & $2-3(F)$ & & 24-h recall & 5.9 & & $88 \cdot 7$ & & $24 \cdot 1$ & & $32 \cdot 0$ & & $8 \cdot 1$ & & & & & & 39.1 & & $10 \cdot 0$ & \\
\hline \multirow[t]{2}{*}{ Belgium ${ }^{(37)}$} & 2014-2015 & 3-5 (M) & 230 & 24-h recall & $6 \cdot 0$ & & $102 \cdot 0$ & & $28 \cdot 0$ & & & & & & & & & & & & & \\
\hline & & $3-5(F)$ & 224 & 24-h recall & $5 \cdot 6$ & & 98.0 & & $27 \cdot 0$ & & & & & & & & & & & & & \\
\hline Chile(38) & 2010 & $2-5$ & 257 & 24-h recall & & & 74.4 & & & & & & & & & & & & & & & \\
\hline \multirow{10}{*}{ Denmark*(39) } & $2006-2007$ & $6-7$ months $(M)$ & 157 & 7-d record & 3.5 & 0.8 & & & & & 4.4 & 5.0 & $2 \cdot 1$ & $2 \cdot 2$ & & & & & & & & \\
\hline & & 8-9 months (M) & 153 & 7-d record & 3.8 & 0.9 & & & & & 5.9 & $6 \cdot 3$ & $2 \cdot 6$ & 2.4 & & & & & & & & \\
\hline & & $10-11$ months $(\mathrm{M})$ & 114 & 7-d record & $4 \cdot 2$ & 0.9 & & & & & $10 \cdot 0$ & 11.0 & $4 \cdot 1$ & 4.0 & & & & & & & & \\
\hline & & 12-23 months (M) & 221 & 7-d record & 4.7 & 1.0 & & & & & $18 \cdot 0$ & $12 \cdot 0$ & 6.5 & 4.0 & & & & & & & & \\
\hline & & 24-36 months (M) & 228 & 7-d record & $5 \cdot 3$ & 1.0 & & & & & $28 \cdot 0$ & $15 \cdot 0$ & $9 \cdot 0$ & 4.2 & & & & & & & & \\
\hline & & $6-7$ months $(\mathrm{F})$ & 159 & 7-d record & 2.9 & 0.8 & & & & & $2 \cdot 7$ & 3.4 & 1.6 & 1.9 & & & & & & & & \\
\hline & & 8-9 months (F) & 136 & 7-d record & 3.5 & 0.9 & & & & & 5.8 & 5.4 & $2 \cdot 7$ & $2 \cdot 2$ & & & & & & & & \\
\hline & & $10-11$ months $(\mathrm{F})$ & 107 & 7-d record & 3.9 & 0.9 & & & & & 7.6 & 7.0 & $3 \cdot 2$ & $2 \cdot 7$ & & & & & & & & \\
\hline & & $12-23$ months (F) & 253 & 7-d record & 4.5 & 1.0 & & & & & $16 \cdot 0$ & 11.0 & 5.9 & 3.4 & & & & & & & & \\
\hline & & $24-36$ months (F) & 215 & 7-d record & $5 \cdot 0$ & 1.0 & & & & & $26 \cdot 0$ & 11.0 & 8.8 & $3 \cdot 7$ & & & & & & & & \\
\hline \multirow[t]{2}{*}{ Estonia $^{(40)}$} & 2015 & $2-5(M)$ & 277 & 24-h recall & $6 \cdot 0$ & & & & & & & & & & $48 \cdot 0$ & & & & & & & \\
\hline & & $2-5(F)$ & 302 & 24-h recall & $5 \cdot 6$ & & & & & & & & & & $46 \cdot 0$ & & & & & & & \\
\hline Finland $(41)$ & 2003-2005 & $1 \mathrm{BF}(\mathrm{M})$ & 55 & 3-d record & $2 \cdot 8$ & 0.8 & $43 \cdot 0$ & $16 \cdot 0$ & & & & & & & 10.5 & 5.9 & & & & & & \\
\hline & & $1 \mathrm{FF}(\mathrm{M})$ & 257 & 3-d record & 3.9 & 0.7 & $69 \cdot 0$ & $18 \cdot 0$ & & & & & & & $13 \cdot 0$ & 9.4 & & & & & & \\
\hline & & $2(\mathrm{M})$ & 112 & 3-d record & 4.6 & 1.0 & 78.0 & 23.0 & & & & & & & 34.3 & $16 \cdot 9$ & & & & & & \\
\hline & & $3(\mathrm{M})$ & 236 & 3-d record & 5.4 & 1.0 & $90 \cdot 0$ & $27 \cdot 0$ & & & & & & & $41 \cdot 7$ & $18 \cdot 3$ & & & & & & \\
\hline & & $1 \mathrm{BF}(\mathrm{F})$ & 57 & $3-d$ record & $2 \cdot 6$ & 0.8 & $38 \cdot 0$ & $15 \cdot 0$ & & & & & & & $9 \cdot 0$ & $6 \cdot 0$ & & & & & & \\
\hline & & $1 \mathrm{FF}(\mathrm{F})$ & 198 & 3-d record & 3.6 & 0.6 & 64.0 & $16 \cdot 0$ & & & & & & & 11.7 & 8.0 & & & & & & \\
\hline & & $2(F)$ & 118 & 3-d record & 4.5 & 0.9 & $77 \cdot 0$ & $21 \cdot 0$ & & & & & & & 31.5 & 14.7 & & & & & & \\
\hline & & $3(F)$ & 235 & 3-d record & $5 \cdot 1$ & 1.0 & $85 \cdot 0$ & $23 \cdot 0$ & & & & & & & $40 \cdot 3$ & $17 \cdot 0$ & & & & & & \\
\hline France ${ }^{(42)}$ & 2014-2015 & $0-11$ months $(\mathrm{M})$ & 80 & 24-h recall & 3.1 & 1.3 & 65.4 & $25 \cdot 6$ & & & & & & & & & & & & & & \\
\hline & & $0-11$ months $(F)$ & & 24-h recall & $2 \cdot 8$ & 0.5 & $65 \cdot 8$ & 13.5 & & & & & & & & & & & & & & \\
\hline & & $1-3(\mathrm{M})$ & 229 & 24-h recall & $5 \cdot 2$ & 0.8 & 92.4 & $17 \cdot 0$ & & & & & & & & & & & & & & \\
\hline & & $1-3(F)$ & & 24-h recall & 4.9 & 1.1 & $85 \cdot 6$ & $18 \cdot 2$ & & & & & & & & & & & & & & \\
\hline Iceland(43,44) & 2005-2007 & 9 months (M) & 93 & 3-d record & 3.2 & & & & & & 3.4 & 4.2 & & & & & & & & & & \\
\hline & & 12 months (M) & 82 & 3-d record & 3.6 & & & & & & 7.6 & 5.4 & & & & & & & & & & \\
\hline & & 9 months $(F)$ & 102 & 3-d record & 3.0 & & & & & & 3.6 & $5 \cdot 2$ & & & & & & & & & & \\
\hline & & 12 months $(F)$ & 88 & 3-d record & 3.3 & & & & & & $9 \cdot 7$ & $7 \cdot 2$ & & & & & & & & & & \\
\hline & 2007 & 3 & 225 & 3-d record & $5 \cdot 8$ & $1 \cdot 1$ & & & & & $26 \cdot 0$ & $13 \cdot 0$ & & & & & & & & & & \\
\hline Ireland ${ }^{(45)}$ & 2010-2011 & 1 & 126 & 4-d record & 4.2 & 0.9 & $70 \cdot 7$ & $5 \cdot 3$ & $26 \cdot 2$ & 1.3 & & & & & & & & & $25 \cdot 1$ & 3.8 & $9 \cdot 0$ & 1.2 \\
\hline & & 2 & 124 & 4-d record & 4.7 & 1.2 & 74.3 & $5 \cdot 7$ & 24.8 & 1.4 & & & & & & & & & 33.4 & 4.9 & 11.0 & 1.5 \\
\hline & & 3 & 126 & 4-d record & 4.8 & 0.9 & 75.9 & 5.9 & 24.9 & 1.4 & & & & & & & & & 41.4 & $5 \cdot 7$ & $13 \cdot 4$ & 1.7 \\
\hline Israel(46) & 2015-2016 & $2-5(M)$ & & 24-h recall & 6.0 & $2 \cdot 2$ & 68.0 & 38.0 & $19 \cdot 6$ & 8.7 & & & & & & & & & & & & \\
\hline & & $2-5(F)$ & & 24-h recall & 5.5 & 1.9 & $61 \cdot 0$ & $32 \cdot 0$ & $18 \cdot 8$ & $7 \cdot 7$ & & & & & & & & & & & & \\
\hline Italy ${ }^{(47)}$ & 2005-2006 & $0-3$ & 52 & 3-d record & 4.7 & 1.8 & 71.4 & $36 \cdot 0$ & 24.9 & 8.8 & & & & & & & & & & & & \\
\hline Mexico $^{(48)}$ & 2012 & $1-4(\mathrm{M})$ & 2113 & 24-h recall & $5 \cdot 8$ & & & & & & & & $12 \cdot 1$ & & & & & & & & & \\
\hline & & $1-4(F)$ & & 24-h recall & 5.5 & & & & & & & & $12 \cdot 1$ & & & & & & & & & \\
\hline Netherlands ${ }^{(49)}$ & 2012-2016 & 1-3 (M) & 332 & 24-h recall & 5.5 & & $102 \cdot 0$ & & 30.7 & & $45 \cdot 8$ & & $12 \cdot 7$ & & & & & & 54.9 & & $15 \cdot 6$ & \\
\hline & & $1-3(F)$ & 340 & 24-h recall & $5 \cdot 2$ & & 98.0 & & 31.4 & & $47 \cdot 2$ & & $14 \cdot 1$ & & & & & & 57.6 & & 17.4 & \\
\hline
\end{tabular}


Children $(<4$ years). For infants and very young children ( $<4$ years), sugar intake data are available for 16 countries: Australia, Belgium, Chile, Denmark, Estonia, Finland, France, Iceland, Ireland, Israel, Italy, Mexico, the Netherlands, Norway, the UK and the United States (Table 3). These include intakes of total sugars (nine countries), added sugars (seven countries), free sugars (four countries) and sucrose (two countries).

Total sugar intake expressed as a percentage of total energy (\%) ranged from $18.8 \%$ for 2-5-year-old girls in Israel to $31.4 \%$ for 1-3-year-old girls in the Netherlands. Intake of added sugars (\%) ranged from $1.5 \%$ in 6-11-month-old girls in the United States to $14.1 \%$ in 1-3-year-old girls in the Netherlands. Intake of free sugars (\%) ranged from $9 \%$ in 1-year-olds in Ireland to $17.4 \%$ in 1-3-year-old girls in the Netherlands.

Children (4-12 years). For children aged 4-12 years, sugar intake data are available for 25 countries: Australia, Austria, Belgium, Brazil, Canada, Chile, China, Denmark, Estonia, Finland, France, Germany, Iceland, India, Ireland, Israel, Italy, Mexico, the Netherlands, New Zealand, Norway, Portugal, Spain, the UK and the United States (Table 4). These include intakes of total sugars (eighteen countries), added sugars (eleven countries), free sugars (six countries), sucrose (four countries) and intrinsic sugars (Spain only).

Total sugar intake (\%) ranged from $8 \%$ in 4-13-year-olds in China to $28.6 \%$ in 4-8-year-old girls in the Netherlands. Intakes of added sugars (\%) ranged from $3 \%$ in 4-13-year-old children in China to $17.0 \%$ in 4-8-year-old girls in the Netherlands. Intakes of free sugars (\%) ranged from $9 \cdot 1 \%$ in 3-month-oldto-10-year-old boys in Portugal to $19.5 \%$ for $4-8$-year-old girls in the Netherlands.

Adolescents (13-18 years). For older children (13-18 years), sugar intake data were available for 24 countries: Andorra, Australia, Austria, Belgium, Brazil, Canada, Chile, Denmark, Estonia, France, Germany, Iceland, India, Ireland, Israel, Italy, Mexico, the Netherlands, New Zealand, Portugal, Slovenia, Spain, the UK and the United States (Table 5). These include intakes of total sugars (sixteen countries), added sugars (twelve countries), free sugars (eight countries), sucrose (four countries) and intrinsic sugars (Spain only).

Total sugar intake (\%E) ranged from $15.4 \%$ in 10-18-year-old boys in Italy to $30 \%$ in 15-16-year-old girls in Slovenia. Intakes of added sugars (\%) ranged from $9 \cdot 1 \%$ in 10-17-year-old girls in Portugal to $16.2 \%$ in 9-18-year-old children in the United States. Intakes of free sugars (\%E) ranged from $9 \cdot 1 \%$ in 10-17-year-old boys in Portugal to $17.9 \%$ for 9-13-year-old boys in the Netherlands.

Adults (19-64 years). For adults, sugar intake data are available for 29 countries: Andorra, Australia, Austria, Belgium, Brazil, Canada, Chile, Denmark, Estonia, Finland, France, Germany, Greece, Hungary, Iceland, India, Ireland, Israel, Italy, Lithuania, Mexico, the Netherlands, New Zealand, Norway, Portugal, Spain, Switzerland, the UK and the United States (Table 6). These include intakes of total sugars (eighteen countries), added sugars (fifteen countries), free sugars (eleven countries), sucrose (four countries) and intrinsic sugars (Spain only). 
NS Nutrition Research Reviews

Table 4. Energy and sugar intake of children (4-12 years)

\begin{tabular}{|c|c|c|c|c|c|c|c|c|c|c|c|c|c|c|c|c|c|c|c|c|c|c|}
\hline \multirow[b]{2}{*}{ Country } & \multirow[b]{2}{*}{ Assessment year } & \multirow[b]{2}{*}{ Age range (years) } & \multirow[b]{2}{*}{$n$} & \multirow[b]{2}{*}{ Method } & \multicolumn{2}{|c|}{ Energy } & \multicolumn{4}{|c|}{ Total sugars } & \multicolumn{4}{|c|}{ Added sugars } & \multicolumn{4}{|c|}{ Sucrose } & \multicolumn{4}{|c|}{ Free sugars } \\
\hline & & & & & MJ & SD & g & SD & $\% \mathrm{E}$ & SD & g & SD & $\% \mathrm{E}$ & SD & $\mathrm{g}$ & SD & $\% \mathrm{E}$ & SD & $\mathrm{g}$ & SD & $\% \mathrm{E}$ & SD \\
\hline \multirow[t]{4}{*}{ Australia ${ }^{(35,36)}$} & 2011-2012 & 4-8 (M) & & 24-h recall & 7.6 & & $110 \cdot 7$ & & $23 \cdot 3$ & & $52 \cdot 2$ & & $10 \cdot 8$ & & & & & & $60 \cdot 4$ & & 12.5 & \\
\hline & & 9-13 (M) & & & $9 \cdot 2$ & & $125 \cdot 4$ & & 21.7 & & 66.9 & & 11.4 & & & & & & $75 \cdot 3$ & & $12 \cdot 9$ & \\
\hline & & $4-8(F)$ & & & $6 \cdot 4$ & & 94.8 & & 23.8 & & 43.5 & & $10 \cdot 5$ & & & & & & $50 \cdot 7$ & & $12 \cdot 4$ & \\
\hline & & $9-13(\mathrm{~F})$ & & & 8.0 & & $115 \cdot 3$ & & 23.5 & & $61 \cdot 0$ & & $12 \cdot 3$ & & & & & & 68.2 & & $13 \cdot 8$ & \\
\hline \multirow[t]{4}{*}{ Austria $^{(56)}$} & 2012 & 7-9 (M) & 67 & 3-d record & $8 \cdot 0$ & & & & & & & & & & & & $12 \cdot 0$ & & & & & \\
\hline & & $10-12(\mathrm{M})$ & 83 & & $8 \cdot 1$ & & & & & & & & & & & & $10 \cdot 0$ & & & & & \\
\hline & & $7-9(\mathrm{~F})$ & 57 & & 8.0 & & & & & & & & & & & & 11.0 & & & & & \\
\hline & & $10-12(\mathrm{~F})$ & 81 & & $7 \cdot 2$ & & & & & & & & & & & & 11.0 & & & & & \\
\hline \multirow[t]{4}{*}{ Belgium ${ }^{(37)}$} & 2014-2015 & $6-9(M)$ & 279 & 24-h recall & $7 \cdot 4$ & & 113.0 & & $26 \cdot 0$ & & & & & & & & & & & & & \\
\hline & & $10-13(\mathrm{M})$ & 210 & & 8.4 & & 118.0 & & 23.0 & & & & & & & & & & & & & \\
\hline & & $6-9(\mathrm{~F})$ & 259 & & 6.5 & & 97.0 & & $25 \cdot 0$ & & & & & & & & & & & & & \\
\hline & & $10-13(F)$ & 239 & & 6.9 & & 95.0 & & 24.0 & & & & & & & & & & & & & \\
\hline Brazil $^{(57)}$ & 2008-2009 & $10-13(\mathrm{M})$ & 1515 & 24-h recall & $8 \cdot 1$ & $2 \cdot 8$ & & & & & 70.0 & 41.0 & $14 \cdot 0$ & $6 \cdot 0$ & & & & & & & & \\
\hline & & $10-13(F)$ & 1566 & & $7 \cdot 8$ & 2.5 & & & & & $73 \cdot 0$ & 41.0 & $15 \cdot 0$ & $7 \cdot 0$ & & & & & & & & \\
\hline Canada ${ }^{(58)}$ & 2015 & $2-8$ & & 24-h recall & & & $101 \cdot 0$ & & 25.9 & & & & & & & & & & & & & \\
\hline Chile*(38) & 2010 & $6-13$ & 673 & 24-h recall & & & $100 \cdot 2$ & & & & & & & & & & & & & & & \\
\hline & & $6-13(M)$ & & & $8 \cdot 2$ & & & & & & & & & & & & & & & & & \\
\hline & & $6-13(F)$ & & & $6 \cdot 8$ & & & & & & & & & & & & & & & & & \\
\hline China $^{\dagger(59)}$ & 2011 & $4-13$ & 1460 & 24-h recall & $5 \cdot 8$ & 0.1 & 26.0 & 0.6 & 8.0 & 0.1 & 9.0 & 0.3 & 3.0 & 0.1 & & & & & & & & \\
\hline Denmark $^{(60)}$ & $2011-2013$ & $4-5(\mathrm{M})$ & 66 & 7-d record & $7 \cdot 9$ & 1.6 & & & & & $40 \cdot 0$ & $19 \cdot 2$ & 9.0 & 3.7 & & & & & & & & \\
\hline & & 6-9 (M) & 150 & & $8 \cdot 7$ & $1 \cdot 8$ & & & & & 55.0 & $26 \cdot 8$ & $11 \cdot 0$ & 4.6 & & & & & & & & \\
\hline & & $10-13(\mathrm{M})$ & 134 & & 9.6 & $2 \cdot 2$ & & & & & 66.0 & $29 \cdot 7$ & $12 \cdot 0$ & 4.6 & & & & & & & & \\
\hline & & $4-5(F)$ & 64 & & 7.0 & $1 \cdot 3$ & & & & & 39.0 & $19 \cdot 1$ & $9 \cdot 0$ & 4.0 & & & & & & & & \\
\hline & & $6-9(F)$ & 141 & & 8.0 & 1.7 & & & & & $50 \cdot 0$ & $25 \cdot 7$ & $10 \cdot 0$ & 4.4 & & & & & & & & \\
\hline & & $10-13(\mathrm{~F})$ & 135 & & $8 \cdot 1$ & 1.8 & & & & & 55.0 & 29.8 & 11.0 & 4.8 & & & & & & & & \\
\hline Estonia $(40)$ & 2015 & $6-9(M)$ & 168 & 24-h recall & $7 \cdot 7$ & & & & & & & & & & $61 \cdot 0$ & & & & & & & \\
\hline & & $10-13(\mathrm{M})$ & 93 & & $8 \cdot 3$ & & & & & & & & & & $59 \cdot 0$ & & & & & & & \\
\hline & & $6-9(\mathrm{~F})$ & 179 & & $6 \cdot 8$ & & & & & & & & & & $56 \cdot 0$ & & & & & & & \\
\hline & & $10-13(F)$ & 89 & & $6 \cdot 7$ & & & & & & & & & & 55.0 & & & & & & & \\
\hline Finland $^{(41)}$ & 2003-2005 & $4(\mathrm{M})$ & 307 & 3-d record & $5 \cdot 8$ & $1 \cdot 1$ & 97.0 & $26 \cdot 0$ & & & & & & & $46 \cdot 9$ & $18 \cdot 0$ & & & & & & \\
\hline & & 6 (M) & 364 & & $6 \cdot 7$ & $1 \cdot 2$ & $108 \cdot 0$ & 28.0 & & & & & & & 51.5 & 19.9 & & & & & & \\
\hline & & $4(F)$ & 247 & & $5 \cdot 5$ & 1.0 & $92 \cdot 0$ & $22 \cdot 0$ & & & & & & & 43.6 & $16 \cdot 0$ & & & & & & \\
\hline & & $6(F)$ & 349 & & 6.0 & $1 \cdot 1$ & 99.0 & $27 \cdot 0$ & & & & & & & $48 \cdot 7$ & $19 \cdot 3$ & & & & & & \\
\hline France $^{(42)}$ & 2014-2015 & $4-6(M)$ & 454 & 24-h recall & $6 \cdot 6$ & $1 \cdot 7$ & $104 \cdot 1$ & $31 \cdot 2$ & & & & & & & & & & & & & & \\
\hline & & $4-6(F)$ & & & $6 \cdot 1$ & 1.4 & 98.0 & 27.5 & & & & & & & & & & & & & & \\
\hline & & $7-10(M)$ & 643 & & $8 \cdot 1$ & $1 \cdot 8$ & $115 \cdot 2$ & $32 \cdot 8$ & & & & & & & & & & & & & & \\
\hline & & $7-10(\mathrm{~F})$ & & & $7 \cdot 2$ & 1.9 & 104.5 & 34.0 & & & & & & & & & & & & & & \\
\hline Germany ${ }^{*}(61)$ & 2006 & $6(\mathrm{M})$ & 106 & 3-d record & $7 \cdot 2$ & 1.4 & 121.3 & $40 \cdot 0$ & & & & & & & & & & & & & & \\
\hline & & $7-9(\mathrm{M})$ & 321 & & 7.8 & 1.6 & 126.5 & $41 \cdot 0$ & & & & & & & & & & & & & & \\
\hline & & $10-11(\mathrm{M})$ & 199 & & 8.0 & 1.8 & 123.0 & $42 \cdot 0$ & & & & & & & & & & & & & & \\
\hline & & $6(\mathrm{~F})$ & 102 & & $6 \cdot 3$ & $1 \cdot 3$ & $101 \cdot 0$ & $29 \cdot 0$ & & & & & & & & & & & & & & \\
\hline & & $7-9(F)$ & 308 & & $7 \cdot 0$ & 1.4 & 114.7 & 41.0 & & & & & & & & & & & & & & \\
\hline & & $10-11(\mathrm{~F})$ & 198 & & 7.6 & 1.6 & 119.6 & $47 \cdot 0$ & & & & & & & & & & & & & & \\
\hline Iceland $(44,62,63)$ & 2007 & 5 & 231 & 3-d record & $6 \cdot 3$ & 1.2 & & & & & 34.0 & $16 \cdot 0$ & & & & & & & & & & \\
\hline & 2011-2012 & 6 & 162 & 3-d record & 6.5 & 1.4 & & & & & & & $11 \cdot 2$ & & & & & & & & & \\
\hline & 2003-2004 & 9 & 175 & 24-h recall & 8.2 & $1 \cdot 8$ & $125 \cdot 0$ & $42 \cdot 0$ & & & 66.0 & 37.0 & & & & & & & & & & \\
\hline India (rural)(64) & $2011-2012$ & 5-14 (M) & 5265 & 24-h recall & & & & & & & 10.5 & $16 \cdot 3$ & & & & & & & & & & \\
\hline & & $5-14(F)$ & 4965 & & & & & & & & $10 \cdot 1$ & $14 \cdot 1$ & & & & & & & & & & \\
\hline
\end{tabular}


Table 4. (Continued)

\begin{tabular}{|c|c|c|c|c|c|c|c|c|c|c|c|c|c|c|c|c|c|c|c|c|c|c|}
\hline \multirow[b]{2}{*}{ Country } & \multirow[b]{2}{*}{ Assessment year } & \multirow[b]{2}{*}{ Age range (years) } & \multirow[b]{2}{*}{$n$} & \multirow[b]{2}{*}{ Method } & \multicolumn{2}{|c|}{ Energy } & \multicolumn{4}{|c|}{ Total sugars } & \multicolumn{4}{|c|}{ Added sugars } & \multicolumn{4}{|c|}{ Sucrose } & \multicolumn{4}{|c|}{ Free sugars } \\
\hline & & & & & MJ & SD & g & SD & $\% \mathrm{E}$ & SD & g & SD & $\% \mathrm{E}$ & SD & $g$ & SD & $\% \mathrm{E}$ & SD & $g$ & SD & $\% \mathrm{E}$ & SD \\
\hline \multirow{2}{*}{ India (urban) $)^{(64)}$} & 2015-2016 & 5-14 (M) & 4526 & 24-h recall & & & & & & & $12 \cdot 3$ & $13 \cdot 0$ & & & & & & & & & & \\
\hline & & $5-14(F)$ & 3961 & & & & & & & & 11.5 & $13 \cdot 0$ & & & & & & & & & & \\
\hline \multirow{2}{*}{ Ireland ${ }^{(45,65)}$} & $2010-2011$ & 4 & 124 & 4-d record & $5 \cdot 3$ & 1.0 & 83.4 & $6 \cdot 2$ & 24.7 & 1.4 & & & & & & & & & 49.0 & 6.4 & 14.4 & 1.8 \\
\hline & $2017-2018$ & $5-12$ & 600 & 4-d record & 6.3 & 1.2 & 73.7 & 19.4 & $18 \cdot 7$ & 4.0 & & & & & & & & & 38.4 & 14.9 & 9.5 & 3.4 \\
\hline \multirow[t]{2}{*}{ Israel(66) } & 2015-2016 & $6-11(\mathrm{M})$ & & 24-h recall & 6.7 & $2 \cdot 3$ & $67 \cdot 0$ & 39.0 & $17 \cdot 0$ & $8 \cdot 2$ & & & & & & & & & & & & \\
\hline & & $6-11(\mathrm{~F})$ & & & 6.0 & $2 \cdot 1$ & 63.0 & 35.0 & 17.7 & 7.8 & & & & & & & & & & & & \\
\hline Italy ${ }^{(47)}$ & $2005-2006$ & $3-10$ & 193 & 3-d record & 8.0 & $2 \cdot 0$ & 86.0 & $30 \cdot 0$ & $17 \cdot 0$ & $5 \cdot 0$ & & & & & & & & & & & & \\
\hline \multirow[t]{3}{*}{ Mexico ${ }^{\dagger(48)}$} & 2012 & $5-11(\mathrm{M})$ & 2753 & 24-h recall & $7 \cdot 8$ & & & & & & & & 11.7 & & & & & & & & & \\
\hline & & $5-11(\mathrm{~F})$ & & & 7.3 & & & & & & & & $12 \cdot 3$ & & & & & & & & & \\
\hline & & $4-13^{\dagger}$ & 3980 & 24-h recall & 7.7 & 0.1 & 92.0 & 1.5 & $20 \cdot 0$ & 0.3 & 55.0 & 1.1 & 12.0 & 0.2 & & & & & & & & \\
\hline \multirow{2}{*}{ Netherlands ${ }^{(49)}$} & 2012-2016 & 4-8 (M) & 261 & 24-h recall & 7.6 & & 129.0 & & 28.4 & & $81 \cdot 0$ & & $16 \cdot 6$ & & & & & & $92 \cdot 1$ & & $19 \cdot 0$ & \\
\hline & & $4-8(F)$ & 259 & & 6.8 & & $116 \cdot 0$ & & 28.6 & & 71.0 & & $17 \cdot 0$ & & & & & & $80 \cdot 6$ & & 19.5 & \\
\hline \multirow[t]{4}{*}{ New Zealand ${ }^{(67)}$} & 2002 & 5-6 (M) & 383 & 24-h recall & $7 \cdot 6$ & & 110.0 & & $24 \cdot 2$ & & & & & & 58.5 & & & & & & & \\
\hline & & $7-10(\mathrm{M})$ & 738 & & 9.0 & & 130.0 & & 24.2 & & & & & & $70 \cdot 8$ & & & & & & & \\
\hline & & $5-6(F)$ & 309 & & 6.8 & & 105.0 & & $25 \cdot 8$ & & & & & & 55.6 & & & & & & & \\
\hline & & $7-10(\mathrm{~F})$ & 687 & & $7 \cdot 8$ & & $115 \cdot 0$ & & 24.5 & & & & & & 63.6 & & & & & & & \\
\hline \multirow[t]{4}{*}{ Norway ${ }^{(68,69)}$} & 2016 & $4(\mathrm{M})$ & 204 & 1-d record & $6 \cdot 1$ & 1.2 & & & & & 30.0 & $18 \cdot 0$ & 8.6 & 4.6 & & & & & & & & \\
\hline & 2015 & $9-13(\mathrm{M})$ & 332 & & 8.6 & $2 \cdot 7$ & 64.0 & 54.0 & 11.9 & 7.9 & & & & & & & & & & & & \\
\hline & 2016 & $4(F)$ & 195 & & 5.5 & 1.4 & & & & & 28.0 & $16 \cdot 0$ & 8.6 & $4 \cdot 3$ & & & & & & & & \\
\hline & 2015 & $9-13(F)$ & 355 & & $7 \cdot 4$ & $2 \cdot 6$ & 60.0 & $48 \cdot 0$ & $12 \cdot 8$ & $7 \cdot 1$ & & & & & & & & & & & & \\
\hline \multirow[t]{2}{*}{ Portugal ${ }^{(70)}$} & $2015-2016$ & 3 months to 10 years $(\mathrm{M})$ & 1470 & 2-d record & 6.9 & & 98.6 & & $25 \cdot 3$ & & 41.6 & & 8.8 & & & & & & $46 \cdot 2$ & & 9.1 & \\
\hline & & 3 months to 10 years $(F)$ & 1438 & & $6 \cdot 6$ & & 89.2 & & $24 \cdot 3$ & & 34.5 & & 8.5 & & & & & & $39 \cdot 2$ & & 9.6 & \\
\hline \multirow[t]{2}{*}{ Spain $^{\ddagger(71,72)}$} & 2013 & 9-12 (M) & 126 & 3-d record & 8.4 & 1.9 & 93.7 & $35 \cdot 3$ & 18.8 & $5 \cdot 4$ & & & & & & & & & $50 \cdot 5$ & 25.9 & 9.9 & 4.1 \\
\hline & & $9-12(\mathrm{~F})$ & 87 & & 7.9 & 1.6 & 88.4 & $30 \cdot 1$ & $18 \cdot 8$ & $5 \cdot 0$ & & & & & & & & & $45 \cdot 9$ & $20 \cdot 6$ & $9 \cdot 6$ & 3.8 \\
\hline \multirow[t]{2}{*}{$\mathrm{UK}^{(73)}$} & $2014 / 15-2015 / 16$ & 4-10 (M) & 276 & 4-d record & $6 \cdot 3$ & 1.4 & & & & & & & & & & & & & 54.5 & $24 \cdot 4$ & 13.6 & $5 \cdot 4$ \\
\hline & & $4-10(F)$ & 238 & & $5 \cdot 8$ & 1.3 & & & & & & & & & & & & & $49 \cdot 9$ & $23 \cdot 2$ & 13.4 & 4.6 \\
\hline \multirow[t]{4}{*}{ United States ${ }^{\dagger(53,55)}$} & $2017-2018$ & $6-11(\mathrm{M})$ & 383 & 24-h recall & 8.4 & 0.2 & 118.0 & 3.1 & & & & & & & & & & & & & & \\
\hline & & $6-11(F)$ & 412 & & $7 \cdot 8$ & 0.2 & $112 \cdot 0$ & 4.2 & & & & & & & & & & & & & & \\
\hline & 2009-2012 & $2-8$ & 2871 & 24-h recall & & & & & & & $61 \cdot 0$ & & 14.3 & & & & & & & & & \\
\hline & & $4-13$ & 3647 & 24-h recall & $7 \cdot 8$ & 0.1 & 124.0 & 1.5 & $26 \cdot 0$ & 0.3 & $76 \cdot 0$ & 1.2 & $16 \cdot 0$ & 0.2 & & & & & & & $9 \cdot 1$ & \\
\hline
\end{tabular}

M: male; F: female.

yy report median intakes.

Mexico (4-13) and United States report SEM

‡ Spain energy intake from ANIBES (2014). Sugar intake reported from Ruiz et al. (2017). 
Table 5. Energy and sugar intake of adolescents ( $13-18$ years)

\begin{tabular}{|c|c|c|c|c|c|c|c|c|c|c|c|c|c|c|c|c|c|c|c|c|c|c|}
\hline \multirow[b]{2}{*}{ Country } & \multirow[b]{2}{*}{ Assessment year } & \multirow[b]{2}{*}{ Age range (years) } & \multirow[b]{2}{*}{$n$} & \multirow[b]{2}{*}{ Method } & \multicolumn{2}{|c|}{ Energy } & \multicolumn{4}{|c|}{ Total sugars } & \multicolumn{4}{|c|}{ Added sugars } & \multicolumn{4}{|c|}{ Sucrose } & \multicolumn{4}{|c|}{ Free sugars } \\
\hline & & & & & MJ & SD & $\mathrm{g}$ & SD & $\% \mathrm{E}$ & SD & $\mathrm{g}$ & SD & $\% \mathrm{E}$ & SD & $g$ & SD & $\% \mathrm{E}$ & SD & $g$ & SD & $\% \mathrm{E}$ & SD \\
\hline \multirow[t]{2}{*}{ Andorra $^{\dagger(74)}$} & 2004-2005 & $12-24(\mathrm{M})$ & 78 & 24-h recall & $10 \cdot 0$ & 1.7 & 104.8 & $37 \cdot 1$ & $18 \cdot 2$ & 6.6 & & & & & & & & & & & & \\
\hline & & $12-24(F)$ & 64 & & 7.8 & 1.2 & 88.6 & 20.4 & 19.4 & 3.5 & & & & & & & & & & & & \\
\hline \multirow[t]{2}{*}{ Australia $^{(35,36)}$} & $2011-2012$ & $14-18(\mathrm{M})$ & & 24-h recall & $10 \cdot 2$ & & $134 \cdot 1$ & & 21.4 & & 82.2 & & $13 \cdot 0$ & & & & & & $92 \cdot 1$ & & 14.5 & \\
\hline & & $14-18(F)$ & & & $8 \cdot 1$ & & $109 \cdot 2$ & & $21 \cdot 4$ & & $62 \cdot 8$ & & $12 \cdot 0$ & & & & & & $70 \cdot 3$ & & 13.5 & \\
\hline Austria $^{(56)}$ & 2012 & $13-14(\mathrm{M})$ & 19 & 3-d record & 8.6 & & & & & & & & & & & & $10 \cdot 0$ & & & & & \\
\hline & & $13-14(F)$ & 25 & & 7.5 & & & & & & & & & & & & 11.0 & & & & & \\
\hline Belgium ${ }^{(37)}$ & 2014-2015 & $14-17(\mathrm{M})$ & 240 & 24-h recall & $9 \cdot 1$ & & $119 \cdot 0$ & & $21 \cdot 0$ & & & & & & & & & & & & & \\
\hline & & $14-17(\mathrm{~F})$ & 239 & & 7.0 & & 93.0 & & $23 \cdot 0$ & & & & & & & & & & & & & \\
\hline Brazil $^{(57)}$ & 2008-2009 & $14-18(\mathrm{M})$ & 1905 & 24-h recall & $9 \cdot 1$ & $3 \cdot 2$ & & & & & $77 \cdot 0$ & $47 \cdot 0$ & 14.0 & $6 \cdot 0$ & & & & & & & & \\
\hline & & $14-18(F)$ & 1811 & & 8.0 & 2.6 & & & & & 78.0 & $45 \cdot 0$ & $16 \cdot 0$ & $7 \cdot 0$ & & & & & & & & \\
\hline Canada ${ }^{(58,75)}$ & 2015 & $9-18$ & & 24-h recall & & & $115 \cdot 0$ & & 22.5 & & & & & & & & & & & & & \\
\hline Chile ${ }^{(38)}$ & 2010 & $14-18$ & 403 & 24-h recall & & & $121 \cdot 2$ & & & & & & & & & & & & & & & \\
\hline Denmark $^{(60)}$ & 2011-2013 & $14-17(M)$ & 117 & 7-d record & $10 \cdot 3$ & 3.3 & & & & & $69 \cdot 0$ & 39.0 & 11.0 & 5.4 & & & & & & & & \\
\hline & & $14-17(\mathrm{~F})$ & 123 & & 7.4 & $2 \cdot 8$ & & & & & 51.0 & 38.8 & 11.0 & $5 \cdot 2$ & & & & & & & & \\
\hline Estonia $^{(40)}$ & 2015 & $14-17(\mathrm{M})$ & 80 & 24-h recall & $9 \cdot 4$ & & & & & & & & & & $66 \cdot 0$ & & & & & & & \\
\hline & & $14-17(F)$ & 117 & & $6 \cdot 6$ & & & & & & & & & & $51 \cdot 0$ & & & & & & & \\
\hline France $^{(42)}$ & 2014-2015 & $11-14(\mathrm{M})$ & 736 & 24-h recall & 8.7 & $2 \cdot 2$ & $112 \cdot 0$ & 36.9 & & & & & & & & & & & & & & \\
\hline & & $11-14(F)$ & & & $8 \cdot 3$ & $3 \cdot 3$ & $108 \cdot 0$ & $46 \cdot 7$ & & & & & & & & & & & & & & \\
\hline & & $15-17(\mathrm{M})$ & 555 & & $9 \cdot 2$ & 3.0 & $110 \cdot 0$ & $44 \cdot 3$ & & & & & & & & & & & & & & \\
\hline & & $15-17(F)$ & & & $6 \cdot 6$ & $2 \cdot 2$ & 82.9 & 35.3 & & & & & & & & & & & & & & \\
\hline Germany ${ }^{\ddagger}(61,76)$ & 2006 & $12(\mathrm{M})$ & 114 & $\mathrm{FFQ}$ & $10 \cdot 6$ & $3 \cdot 2$ & $167 \cdot 0$ & $71 \cdot 8$ & & & & & & & & & & & & & & \\
\hline & & $13-14(\mathrm{M})$ & 214 & FFQ & 11.7 & 3.8 & $187 \cdot 6$ & 83.1 & & & & & & & & & & & & & & \\
\hline & & $15-17(\mathrm{M})$ & 294 & $\mathrm{FFQ}$ & 14.3 & 5.4 & $221 \cdot 3$ & 113.4 & & & & & & & & & & & & & & \\
\hline & 2005-2006 & $14-80(\mathrm{M})$ & 7093 & 24-h recall & $10 \cdot 9 \neq$ & & $124^{\ddagger}$ & & $19 \cdot 3$ & & & & & & & & & & & & & \\
\hline & & $12(\mathrm{~F})$ & 103 & FFQ & $9 \cdot 3$ & $3 \cdot 2$ & 154.5 & 86.0 & & & & & & & & & & & & & & \\
\hline & & $13-14(F)$ & 230 & $\mathrm{FFQ}$ & 9.5 & $2 \cdot 7$ & $160 \cdot 3$ & $70 \cdot 9$ & & & & & & & & & & & & & & \\
\hline & & $15-17(\mathrm{~F})$ & 317 & $\mathrm{FFQ}$ & $9 \cdot 9$ & 3.8 & $175 \cdot 1$ & $96 \cdot 2$ & & & & & & & & & & & & & & \\
\hline & $2005-2006$ & $15-19(\mathrm{M})$ & 506 & 24-h recall & $9 \cdot 2$ & & & & & & & & & & & & $10 \cdot 8$ & & & & & \\
\hline & & $15-19(\mathrm{~F})$ & 536 & 24-h recall & 6.5 & & & & & & & & & & & & $12 \cdot 8$ & & & & & \\
\hline & 2005-2006 & $14-80(F)$ & 8278 & 24-h recall & $6 \cdot 8^{\ddagger}$ & & $113^{\ddagger}$ & & 23.6 & & & & & & & & & & & & & \\
\hline & $2006-2016$ & 3-18 (M) & 2163 & 3-d record & $7 \cdot 2$ & & & & $25 \cdot 6$ & & & & $12 \cdot 0$ & & & & & & & & $16 \cdot 1$ & \\
\hline & & $3-18(F)$ & 1953 & & 6.4 & & & & $25 \cdot 5$ & & & & 11.8 & & & & & & & & $16 \cdot 0$ & \\
\hline Iceland $(63)$ & 2003-2004 & 15 & 150 & 24-h recall & $10 \cdot 3$ & 2.7 & 167.0 & 68.0 & & & 101.0 & 64.0 & & & & & & & & & & \\
\hline India (rural) $)^{(64)}$ & 2011-2012 & $15-19(\mathrm{M})$ & 2344 & 24-h recall & & & & & & & $12 \cdot 0$ & $16 \cdot 6$ & & & & & & & & & & \\
\hline & & $15-19(\mathrm{~F})$ & 2447 & & & & & & & & 11.8 & $15 \cdot 8$ & & & & & & & & & & \\
\hline India (urban) ${ }^{(64)}$ & 2015-2016 & 15-19 (M) & 2328 & 24-h recall & & & & & & & $13 \cdot 8$ & 14.0 & & & & & & & & & & \\
\hline & & $15-19(F)$ & 2188 & & & & & & & & $12 \cdot 8$ & $13 \cdot 0$ & & & & & & & & & & \\
\hline Ireland( & $2005-2006$ & $13-17$ & 441 & 7-d diary & 8.3 & 2.4 & 108.5 & 43.0 & 20.4 & $5 \cdot 0$ & 65.7 & $32 \cdot 0$ & 12.4 & 4.9 & & & & & & & & \\
\hline Israel|(79) & $2015-2016$ & $12-15(M)$ & 1020 & 24-h recall & $10 \cdot 0$ & 4.5 & $146 \cdot 0$ & 85.0 & & & & & & & & & & & & & & \\
\hline & & $12-15(F)$ & 1165 & & 9.6 & 4.6 & $149 \cdot 0$ & 89.0 & & & & & & & & & & & & & & \\
\hline & & $16-18(\mathrm{M})$ & 767 & & 10.5 & 4.6 & 144.0 & 89.0 & & & & & & & & & & & & & & \\
\hline & & $16-18(\mathrm{~F})$ & 954 & & 9.4 & 4.4 & $142 \cdot 0$ & 85.0 & & & & & & & & & & & & & & \\
\hline Italy ${ }^{(47)}$ & $2005-2006$ & $10-18(\mathrm{M})$ & 108 & 3-d record & $10 \cdot 8$ & $3 \cdot 1$ & $107 \cdot 6$ & 53.7 & $15 \cdot 4$ & 4.7 & & & & & & & & & & & & \\
\hline & & $10-18(\mathrm{~F})$ & 139 & & 8.7 & $2 \cdot 2$ & 88.4 & 35.6 & $15 \cdot 8$ & $5 \cdot 2$ & & & & & & & & & & & & \\
\hline Mexico $^{(48)}$ & 2012 & $12-19(\mathrm{M})$ & 2056 & 24-h recall & $9 \cdot 8$ & & & & & & & & $12 \cdot 7$ & & & & & & & & & \\
\hline & & $12-19(F)$ & & & 7.8 & & & & & & & & 14.4 & & & & & & & & & \\
\hline Netherlands ${ }^{(49)}$ & 2012-2016 & $9-13(\mathrm{M})$ & 259 & 24-h recall & $9 \cdot 2$ & & 137.0 & & $25 \cdot 8$ & & $88 \cdot 4$ & & $15 \cdot 9$ & & & & & & $99 \cdot 3$ & & $17 \cdot 9$ & \\
\hline & & $14-18(\mathrm{M})$ & 270 & & $10 \cdot 1$ & & 138.0 & & $24 \cdot 0$ & & 87.8 & & 14.8 & & & & & & 97.6 & & $16 \cdot 6$ & \\
\hline & & $9-13(\mathrm{~F})$ & 260 & & 7.5 & & 114.0 & & $26 \cdot 0$ & & $70 \cdot 6$ & & $15 \cdot 3$ & & & & & & 79.3 & & 17.4 & \\
\hline & & $14-18(F)$ & 254 & & 7.8 & & 111.0 & & $24 \cdot 3$ & & 66.4 & & 14.0 & & & & & & $75 \cdot 2$ & & $15 \cdot 8$ & \\
\hline
\end{tabular}


Total sugars intake (\%E) ranged from $12.7 \%$ in 35-49-yearold men and women in Lithuania to $26 \%$ in 19-30-year-old women in the Netherlands. Intakes of added sugars (\%E) ranged from $6.3 \%$ in 50-59-year-old men in Norway to $14 \%$ in $19-59$ year-old women in Brazil. Intakes of free sugars (\%E) ranged from $6.9 \%$ in $18-64$-year-old men in Portugal to $18.1 \%$ in $51-$ 64-year-old women in Austria.

Adults (>65 years). For older adults, sugar intake data are available for 26 countries: Andorra, Australia, Austria, Brazil, Chile, Denmark, Estonia, Finland, France, Germany, Greece, Hungary, Iceland, India, Ireland, Israel, Italy, Lithuania, Mexico, the Netherlands, New Zealand, Norway, Portugal, Spain, the UK and the United States (Table 7). These include intakes of total sugars (fifteen countries), added sugars (eleven countries), free sugars (seven countries), sucrose (five countries) and intrinsic sugars (Spain only).

Total sugar intake (\%E) ranged from $13.2 \%$ in 65-75-year-old adults in Lithuania to $23.3 \%$ in women $\geq 71$ years old in New Zealand. Intakes of added sugars (\%E) ranged from $4 \%$ in men aged $65-84$ years in Portugal to $12 \%$ in women $\geq 60$ years old in Brazil. Intakes of free sugars (\%E) ranged from $4.4 \%$ in $65-$ 84-year-old men in Portugal to $12.5 \%$ for men aged $>75$ years old in the UK.

Patterns of dietary sugars intake. Our analysis shows that intakes (expressed as \%E) of both free and added sugars are highest in children and adolescents and are lowest for older adults.

Based on data as reported and one data point per age category per country, crude global estimates of mean intakes of free sugars (\%E) were $12 \%$ for 1-4-year-olds, $12 \%$ for $4-12$-yearolds, $14 \%$ for $13-18$-year-olds, $11 \%$ for 19-64-year-olds and 8 $\%$ for $>65$-year-olds. For added sugars, crude global estimates of mean intakes were $8 \%$ for 1-4-year-olds, $11 \%$ for $4-12$ year-olds, $13 \%$ for 13 -18-year-olds, $10 \%$ for $19-64$-year-olds and $8 \%$ for $>65$-year-olds. These findings indicate that, where quantified, intakes of both added and free sugars are generally higher than recommended dietary guidelines at all life stages.

Such patterns of added sugars intake across the lifecycle are similar to previous findings, with the highest intakes in schoolaged children and adolescents and the lower intakes observed in young children and adults (particularly older adults) ${ }^{(15,97)}$. Findings from individual countries such as Australia, Canada, the UK and Ireland have shown some reduction in intakes of added/free sugars in children and adolescents over the last 15-20 years ${ }^{(35,73,75,98)}$. For example, free sugar intake in Ireland has decreased from 16\% TE (2003-2004) to 9\%TE $(2017-2018)^{(98)}$. These studies have suggested that this reduction is largely due to reformulation, particularly in the sweetened beverage categories which are commonly consumed by children and adolescents. It is difficult to draw stronger conclusions beyond this due to differing terminology and methodologies used between countries and over time.

In brief, two observations are apparent from this analysis of published dietary sugars intake data globally. The first is the high intake of added/free sugars compared with recommendations at all life stages but particularly in school-aged children and 
Table 6. Energy and sugar intake of adults (19-64 years)

\begin{tabular}{|c|c|c|c|c|c|c|c|c|c|c|c|c|c|c|c|c|c|c|c|c|c|c|}
\hline \multirow[b]{2}{*}{ Country } & \multirow[b]{2}{*}{ Assessment year } & \multirow[b]{2}{*}{ Age range (years) } & \multirow[b]{2}{*}{$n$} & \multirow[b]{2}{*}{ Method } & \multicolumn{2}{|c|}{ Energy } & \multicolumn{4}{|c|}{ Total sugars } & \multicolumn{4}{|c|}{ Added sugars } & \multicolumn{4}{|c|}{ Sucrose } & \multicolumn{4}{|c|}{ Free sugars } \\
\hline & & & & & MJ & SD & g & SD & $\% \mathrm{E}$ & SD & g & $\mathrm{SD}$ & $\% \mathrm{E}$ & SD & g & $\mathrm{SD}$ & $\% \mathrm{E}$ & SD & g & SD & $\% \mathrm{E}$ & SD \\
\hline \multirow[t]{4}{*}{ Andorra $^{\dagger(74)}$} & 2004-2005 & 25-44 (M) & 222 & 24-h recall & 8.8 & $1 \cdot 2$ & 88.4 & $15 \cdot 8$ & 17.5 & $2 \cdot 4$ & & & & & & & & & & & & \\
\hline & & 45-64 (M) & 85 & & 8.0 & 1.2 & 83.7 & 11.6 & $18 \cdot 2$ & $2 \cdot 2$ & & & & & & & & & & & & \\
\hline & & $25-44(F)$ & 215 & & 6.9 & 1.4 & $75 \cdot 1$ & 18.9 & 18.4 & 3.5 & & & & & & & & & & & & \\
\hline & & $45-64(F)$ & 143 & & 6.8 & $1 \cdot 3$ & 76.8 & $18 \cdot 8$ & $19 \cdot 1$ & $4 \cdot 1$ & & & & & & & & & & & & \\
\hline \multirow{6}{*}{ Australia $^{(35,36)}$} & 2011-2012 & $19-30(\mathrm{M})$ & & 24-h recall & 11.0 & & 131.0 & & $19 \cdot 1$ & & $75 \cdot 5$ & & $10 \cdot 8$ & & & & & & 85.4 & & $12 \cdot 3$ & \\
\hline & & $31-50$ (M) & & & $10 \cdot 2$ & & $119 \cdot 4$ & & $18 \cdot 9$ & & 61.9 & & 9.4 & & & & & & 69.9 & & $10 \cdot 7$ & \\
\hline & & 51-70 (M) & & & 9.3 & & 101.0 & & $17 \cdot 3$ & & $46 \cdot 8$ & & 7.8 & & & & & & $54 \cdot 1$ & & 9.0 & \\
\hline & & $19-30(\mathrm{~F})$ & & & 7.9 & & 99.0 & & 20.5 & & $53 \cdot 1$ & & $10 \cdot 8$ & & & & & & $60 \cdot 4$ & & $12 \cdot 3$ & \\
\hline & & $31-50(F)$ & & & 7.5 & & 91.1 & & $19 \cdot 7$ & & $42 \cdot 5$ & & 8.9 & & & & & & 48.5 & & $10 \cdot 2$ & \\
\hline & & $51-70(\mathrm{~F})$ & & & 7.3 & & 87.0 & & $19 \cdot 2$ & & $36 \cdot 8$ & & $7 \cdot 7$ & & & & & & $42 \cdot 0$ & & 8.8 & \\
\hline \multirow[t]{6}{*}{ Austria $^{(83)}$} & 2017 & $19-24(\mathrm{M})$ & 89 & 24-h recall & 11.1 & 3.4 & & & & & & & & & & & & & & & $16 \cdot 4$ & 8.1 \\
\hline & & 25-50 (M) & 478 & & $10 \cdot 2$ & $2 \cdot 8$ & & & & & & & & & & & & & & & $16 \cdot 4$ & 7.4 \\
\hline & & 51-64 (M) & 169 & & $10 \cdot 0$ & $2 \cdot 7$ & & & & & & & & & & & & & & & $16 \cdot 8$ & $7 \cdot 0$ \\
\hline & & 19-24 (F) & 181 & & 7.6 & $2 \cdot 3$ & & & & & & & & & & & & & & & $16 \cdot 8$ & 7.6 \\
\hline & & $25-50(F)$ & 856 & & 7.6 & $2 \cdot 2$ & & & & & & & & & & & & & & & $17 \cdot 5$ & 7.0 \\
\hline & & $51-64(\mathrm{~F})$ & 245 & & 7.5 & $2 \cdot 2$ & & & & & & & & & & & & & & & $18 \cdot 1$ & 6.8 \\
\hline Belgium $^{(37)}$ & 2014-2015 & 18-39 (M) & 205 & 24-h recall & 9.8 & & 113.0 & & $19 \cdot 0$ & & & & & & & & & & & & & \\
\hline & & 40-64 (M) & 284 & & $9 \cdot 3$ & & 99.0 & & $18 \cdot 0$ & & & & & & & & & & & & & \\
\hline & & $18-39(F)$ & 315 & & 7.1 & & 87.0 & & $20 \cdot 0$ & & & & & & & & & & & & & \\
\hline & & $40-64(\mathrm{~F})$ & 322 & & 6.7 & & 77.0 & & 19.0 & & & & & & & & & & & & & \\
\hline Brazil $^{(57)}$ & 2008-2009 & $19-59(\mathrm{M})$ & 10287 & 24-h recall & 8.9 & $3 \cdot 0$ & & & & & $67 \cdot 0$ & $41 \cdot 0$ & $13 \cdot 0$ & $6 \cdot 0$ & & & & & & & & \\
\hline & & $19-59(\mathrm{~F})$ & 11344 & & $7 \cdot 2$ & $2 \cdot 3$ & & & & & $61 \cdot 0$ & $36 \cdot 0$ & 14.0 & $6 \cdot 0$ & & & & & & & & \\
\hline Canada $\neq^{(58,75)}$ & 2015 & $19+(\mathrm{M})$ & & 24-h recall & & & 94.0 & & $17 \cdot 7$ & & & & & & & & & & & & & \\
\hline & & $19+(F)$ & & & & & $75 \cdot 0$ & & $18 \cdot 8$ & & & & & & & & & & & & & \\
\hline & & $19+$ & 11817 & 24-h recall & & & 86.9 & 1.0 & $18 \cdot 8$ & 0.2 & 41.7 & 0.8 & 8.6 & $0 \cdot 1$ & & & & & 47.5 & 0.9 & 9.9 & 0.1 \\
\hline Chile ${ }^{\S(38)}$ & 2010 & $19-29$ & 660 & 24-h recall & & & 89.1 & & & & & & & & & & & & & & & \\
\hline & & $30-49$ & 1076 & & & & 71.2 & & & & & & & & & & & & & & & \\
\hline & & $50-64$ & 945 & & & & $52 \cdot 3$ & & & & & & & & & & & & & & & \\
\hline & & $14-64(\mathrm{M})$ & & & 9.4 & & & & & & & & & & & & & & & & & \\
\hline & & $14-64(F)$ & & & 6.7 & & & & & & & & & & & & & & & & & \\
\hline Denmark $^{(60)}$ & $2011-2013$ & $18-24(\mathrm{M})$ & 170 & 7-d record & $10 \cdot 7$ & 3.4 & & & & & $61 \cdot 0$ & 47.4 & $10 \cdot 0$ & 6.5 & & & & & & & & \\
\hline & & 25-34 (M) & 190 & & 11.6 & 3.7 & & & & & 68.0 & $46 \cdot 8$ & $10 \cdot 0$ & $5 \cdot 7$ & & & & & & & & \\
\hline & & 35-44 (M) & 253 & & 11.4 & $2 \cdot 9$ & & & & & 63.0 & $49 \cdot 2$ & $9 \cdot 0$ & $5 \cdot 7$ & & & & & & & & \\
\hline & & $45-54(\mathrm{M})$ & 297 & & 11.4 & 3.3 & & & & & 53.0 & $45 \cdot 9$ & $7 \cdot 0$ & 4.9 & & & & & & & & \\
\hline & & 55-64 (M) & 292 & & 11.0 & 3.0 & & & & & $46 \cdot 0$ & 34.6 & $7 \cdot 0$ & 4.7 & & & & & & & & \\
\hline & & $18-24(F)$ & 176 & & 8.1 & 2.5 & & & & & $52 \cdot 0$ & $40 \cdot 0$ & 11.0 & 6.9 & & & & & & & & \\
\hline & & $25-34(F)$ & 185 & & 8.8 & 2.9 & & & & & $50 \cdot 0$ & 34.2 & 9.0 & 4.8 & & & & & & & & \\
\hline & & $35-44(F)$ & 289 & & 8.8 & $2 \cdot 2$ & & & & & $47 \cdot 0$ & 32.0 & 9.0 & $5 \cdot 3$ & & & & & & & & \\
\hline & & $45-54(F)$ & 318 & & 8.5 & $2 \cdot 1$ & & & & & $42 \cdot 0$ & $27 \cdot 6$ & 8.0 & $5 \cdot 0$ & & & & & & & & \\
\hline & & $55-64(\mathrm{~F})$ & 322 & & 8.3 & $2 \cdot 1$ & & & & & 33.0 & 21.4 & $7 \cdot 0$ & $3 \cdot 6$ & & & & & & & & \\
\hline Estonia ${ }^{(40)}$ & 2015 & 18-24 (M) & 135 & 24-h recall & $9 \cdot 7$ & & & & & & & & & & 58.0 & & & & & & & \\
\hline & & $25-29(\mathrm{M})$ & 77 & & 9.5 & & & & & & & & & & $55 \cdot 0$ & & & & & & & \\
\hline & & 30-34 (M) & 85 & & 8.6 & & & & & & & & & & $52 \cdot 0$ & & & & & & & \\
\hline & & 35-39 (M) & 84 & & 9.5 & & & & & & & & & & 55.0 & & & & & & & \\
\hline & & 40-44 (M) & 69 & & 8.7 & & & & & & & & & & $45 \cdot 0$ & & & & & & & \\
\hline & & 45-49 (M) & 67 & & 8.7 & & & & & & & & & & $47 \cdot 0$ & & & & & & & \\
\hline & & $50-54(\mathrm{M})$ & 73 & & 8.9 & & & & & & & & & & $46 \cdot 0$ & & & & & & & \\
\hline & & $55-59(\mathrm{M})$ & 75 & & 8.2 & & & & & & & & & & 44.0 & & & & & & & \\
\hline
\end{tabular}


Nutrition Research Reviews

Table 6. (Continued)

\begin{tabular}{|c|c|c|c|c|c|c|c|c|c|c|c|c|c|c|c|c|c|c|c|c|c|c|}
\hline \multirow[b]{2}{*}{ Country } & \multirow[b]{2}{*}{ Assessment year } & \multirow[b]{2}{*}{ Age range (years) } & \multirow[b]{2}{*}{$n$} & \multirow[b]{2}{*}{ Method } & \multicolumn{2}{|c|}{ Energy } & \multicolumn{4}{|c|}{ Total sugars } & \multicolumn{4}{|c|}{ Added sugars } & \multicolumn{4}{|c|}{ Sucrose } & \multicolumn{4}{|c|}{ Free sugars } \\
\hline & & & & & $\mathrm{MJ}$ & SD & g & SD & $\% \mathrm{E}$ & SD & $\mathrm{g}$ & SD & $\% \mathrm{E}$ & SD & $\mathrm{g}$ & SD & $\% \mathrm{E}$ & SD & $\mathrm{g}$ & SD & $\% \mathrm{E}$ & $\mathrm{SD}$ \\
\hline & & $18-24(F)$ & 192 & & 6.8 & & & & & & & & & & 48.0 & & & & & & & \\
\hline & & $25-29(\mathrm{~F})$ & 137 & & 7.6 & & & & & & & & & & 54.0 & & & & & & & \\
\hline & & $30-34(F)$ & 158 & & $7 \cdot 4$ & & & & & & & & & & 49.0 & & & & & & & \\
\hline & & 35-39 (F) & 160 & & $7 \cdot 2$ & & & & & & & & & & 48.0 & & & & & & & \\
\hline & & $40-44(F)$ & 167 & & $6 \cdot 4$ & & & & & & & & & & 41.0 & & & & & & & \\
\hline & & $45-49(F)$ & 168 & & $6 \cdot 2$ & & & & & & & & & & 38.0 & & & & & & & \\
\hline & & $50-54(\mathrm{~F})$ & 136 & & 6.3 & & & & & & & & & & $42 \cdot 0$ & & & & & & & \\
\hline & & $55-59(\mathrm{~F})$ & 160 & & 6.4 & & & & & & & & & & 38.0 & & & & & & & \\
\hline \multirow[t]{6}{*}{ Finland ${ }^{(84)}$} & 2017 & $18-24(\mathrm{M})$ & 47 & 24-h recall & 10.9 & & & & & & & & & & 37.0 & & $6 \cdot 0$ & & & & & \\
\hline & & 25-44 (M) & 221 & & $10 \cdot 1$ & & & & & & & & & & $47 \cdot 0$ & & $7 \cdot 8$ & & & & & \\
\hline & & 45-64 (M) & 308 & & 9.1 & & & & & & & & & & 43.0 & & 8.1 & & & & & \\
\hline & & $18-24(F)$ & 52 & & $7 \cdot 0$ & & & & & & & & & & 38.0 & & $9 \cdot 3$ & & & & & \\
\hline & & $25-44(F)$ & 259 & & $7 \cdot 7$ & & & & & & & & & & 39.0 & & 8.5 & & & & & \\
\hline & & $45-64(F)$ & 317 & & 7.5 & & & & & & & & & & 41.0 & & $9 \cdot 3$ & & & & & \\
\hline \multirow{4}{*}{ France $^{(42)}$} & 2014-2015 & $18-44$ (M) & 1287 & 24-h recall & 10.6 & 2.9 & $119 \cdot 2$ & $44 \cdot 1$ & & & & & & & & & & & & & & \\
\hline & & $18-44(F)$ & & & 7.8 & $2 \cdot 4$ & 89.6 & $38 \cdot 7$ & & & & & & & & & & & & & & \\
\hline & & 45-64 (M) & 1134 & & 10.4 & $3 \cdot 2$ & 99.8 & 44.6 & & & & & & & & & & & & & & \\
\hline & & $45-64(F)$ & & & 7.4 & $2 \cdot 6$ & 79.9 & $41 \cdot 3$ & & & & & & & & & & & & & & \\
\hline \multirow[t]{10}{*}{ Germany ${ }^{\S(85)}$} & 2005-2006 & $19-25(\mathrm{M})$ & 469 & 24-h recall & 9.5 & & & & & & & & & & & & $10 \cdot 7$ & & & & & \\
\hline & & 25-35 (M) & 614 & & 9.5 & & & & & & & & & & & & $10 \cdot 4$ & & & & & \\
\hline & & $35-51(M)$ & 1946 & & 9.9 & & & & & & & & & & & & 9.9 & & & & & \\
\hline & & $51-65(M)$ & 1460 & & 9.3 & & & & & & & & & & & & 8.8 & & & & & \\
\hline & & $19-25(F)$ & 486 & & 7.0 & & & & & & & & & & & & $12 \cdot 8$ & & & & & \\
\hline & & $25-35(F)$ & 852 & & $7 \cdot 3$ & & & & & & & & & & & & $12 \cdot 3$ & & & & & \\
\hline & & $35-51(\mathrm{~F})$ & 2648 & & 7.3 & & & & & & & & & & & & 11.5 & & & & & \\
\hline & & $51-65(\mathrm{~F})$ & 1740 & & $7 \cdot 0$ & & & & & & & & & & & & $11 \cdot 1$ & & & & & \\
\hline & 2005-2007 & 15-80 (M) & & 24-h recall & & & 120.0 & & $20 \cdot 0$ & & & & & & & & & & $78 \cdot 0$ & & $13 \cdot 0$ & \\
\hline & & $15-80(\mathrm{~F})$ & & & & & $107 \cdot 0$ & & $24 \cdot 0$ & & & & & & & & & & $61 \cdot 0$ & & 14.0 & \\
\hline Greece ${ }^{\|(86)}$ & 1995-2000 & 35-44 (M) & 1311 & 24-h recall & & & 87.3 & & & & & & & & & & & & & & & \\
\hline & & 45-54 (M) & & & & & $85 \cdot 4$ & & & & & & & & & & & & & & & \\
\hline & & 55-64 (M) & & & & & 98.2 & & & & & & & & & & & & & & & \\
\hline & & $35-44(F)$ & 1373 & & & & 85.1 & & & & & & & & & & & & & & & \\
\hline & & $45-54(F)$ & & & & & $87 \cdot 4$ & & & & & & & & & & & & & & & \\
\hline & & $55-64(F)$ & & & & & 88.5 & & & & & & & & & & & & & & & \\
\hline Hungary $\mathbb{q}(87)$ & 2014 & 18-34 (M) & 103 & 3-d record & 12.4 & & & & & & & & 9.3 & & & & & & & & & \\
\hline & & 35-64 (M) & 199 & & $11 \cdot 3$ & & & & & & & & $7 \cdot 3$ & & & & & & & & & \\
\hline & & $18-34(F)$ & 112 & & 8.9 & & & & & & & & $11 \cdot 3$ & & & & & & & & & \\
\hline & & 35-64 (F) & 263 & & 8.5 & & & & & & & & 8.0 & & & & & & & & & \\
\hline Iceland(88) & 2010-2011 & $18-30(\mathrm{M})$ & 131 & 24-h recall & 11.0 & 3.7 & 129.0 & 54.0 & & & $76 \cdot 0$ & $47 \cdot 0$ & & & & & & & & & & \\
\hline & & $31-60(\mathrm{M})$ & 350 & & $10 \cdot 0$ & $3 \cdot 1$ & $105 \cdot 0$ & $57 \cdot 0$ & & & $56 \cdot 0$ & $47 \cdot 0$ & & & & & & & & & & \\
\hline & & $18-30(\mathrm{~F})$ & 119 & & 7.9 & $2 \cdot 1$ & 108.0 & $47 \cdot 0$ & & & $55 \cdot 0$ & 41.0 & & & & & & & & & & \\
\hline & & $31-60(\mathrm{~F})$ & 394 & & 7.5 & $2 \cdot 2$ & 86.0 & $42 \cdot 0$ & & & $40 \cdot 0$ & $32 \cdot 0$ & & & & & & & & & & \\
\hline India (rural) ${ }^{(64)}$ & 2011-2012 & 20-24 (M) & 2031 & 24-h recall & & & & & & & $12 \cdot 9$ & $18 \cdot 2$ & & & & & & & & & & \\
\hline & & $25-29(\mathrm{M})$ & 2011 & & & & & & & & 11.4 & 13.8 & & & & & & & & & & \\
\hline & & 30-34 (M) & 1875 & & & & & & & & $12 \cdot 5$ & 21.4 & & & & & & & & & & \\
\hline & & $35-39(\mathrm{M})$ & 1800 & & & & & & & & $12 \cdot 1$ & $16 \cdot 3$ & & & & & & & & & & \\
\hline & & $40-44$ (M) & 1539 & & & & & & & & 11.9 & $15 \cdot 7$ & & & & & & & & & & \\
\hline & & 45-49 (M) & 1511 & & & & & & & & $13 \cdot 3$ & $18 \cdot 4$ & & & & & & & & & & \\
\hline
\end{tabular}


Nutrition Research Reviews

Table 6. (Continued)

\begin{tabular}{|c|c|c|c|c|c|c|c|c|c|c|c|c|c|c|c|c|c|c|c|c|c|c|}
\hline \multirow[b]{2}{*}{ Country } & \multirow[b]{2}{*}{ Assessment year } & \multirow[b]{2}{*}{ Age range (years) } & \multirow[b]{2}{*}{$n$} & \multirow[b]{2}{*}{ Method } & \multicolumn{2}{|c|}{ Energy } & \multicolumn{4}{|c|}{ Total sugars } & \multicolumn{4}{|c|}{ Added sugars } & \multicolumn{4}{|c|}{ Sucrose } & \multicolumn{4}{|c|}{ Free sugars } \\
\hline & & & & & MJ & SD & g & SD & $\% \mathrm{E}$ & SD & g & SD & $\% \mathrm{E}$ & SD & $g$ & SD & $\% \mathrm{E}$ & SD & g & SD & $\% \mathrm{E}$ & SD \\
\hline & & 50-59 (M) & 2106 & & & & & & & & $13 \cdot 8$ & 20.5 & & & & & & & & & & \\
\hline & & $60-69(\mathrm{M})$ & 1578 & & & & & & & & 14.4 & 18.7 & & & & & & & & & & \\
\hline & & $20-24(F)$ & 2367 & & & & & & & & $12 \cdot 1$ & $15 \cdot 2$ & & & & & & & & & & \\
\hline & & $25-29(\mathrm{~F})$ & 2330 & & & & & & & & $12 \cdot 8$ & $17 \cdot 4$ & & & & & & & & & & \\
\hline & & $30-34(F)$ & 1890 & & & & & & & & $12 \cdot 0$ & 14.5 & & & & & & & & & & \\
\hline & & $35-39(F)$ & 2015 & & & & & & & & $12 \cdot 9$ & $16 \cdot 0$ & & & & & & & & & & \\
\hline & & $40-44(\mathrm{~F})$ & 1541 & & & & & & & & $13 \cdot 6$ & $17 \cdot 7$ & & & & & & & & & & \\
\hline & & $45-49(\mathrm{~F})$ & 1667 & & & & & & & & 13.5 & $15 \cdot 0$ & & & & & & & & & & \\
\hline & & $50-59(\mathrm{~F})$ & 2125 & & & & & & & & 13.4 & $15 \cdot 7$ & & & & & & & & & & \\
\hline & & $60-69(\mathrm{~F})$ & 1528 & & & & & & & & $13 \cdot 6$ & 17.4 & & & & & & & & & & \\
\hline \multirow{16}{*}{ India (urban) $)^{(64)}$} & 2015-2016 & $20-24(\mathrm{M})$ & 2049 & 24-h recall & & & & & & & 14.0 & $13 \cdot 6$ & & & & & & & & & & \\
\hline & & $25-29(\mathrm{M})$ & 1830 & & & & & & & & 14.5 & 13.4 & & & & & & & & & & \\
\hline & & 30-34 (M) & 1877 & & & & & & & & $14 \cdot 3$ & $12 \cdot 8$ & & & & & & & & & & \\
\hline & & 35-39 (M) & 1961 & & & & & & & & 14.4 & $15 \cdot 0$ & & & & & & & & & & \\
\hline & & $40-44(\mathrm{M})$ & 1935 & & & & & & & & 15.4 & 14.0 & & & & & & & & & & \\
\hline & & 45-49 (M) & 1803 & & & & & & & & $15 \cdot 6$ & $15 \cdot 0$ & & & & & & & & & & \\
\hline & & 50-59 (M) & 2641 & & & & & & & & $16 \cdot 6$ & $16 \cdot 0$ & & & & & & & & & & \\
\hline & & 60-69 (M) & 1366 & & & & & & & & $16 \cdot 5$ & $15 \cdot 0$ & & & & & & & & & & \\
\hline & & $20-24(F)$ & 2139 & & & & & & & & 14.7 & 14.0 & & & & & & & & & & \\
\hline & & $25-29(\mathrm{~F})$ & 2163 & & & & & & & & $15 \cdot 6$ & 14.0 & & & & & & & & & & \\
\hline & & 30-34 (F) & 2177 & & & & & & & & $16 \cdot 8$ & $15 \cdot 0$ & & & & & & & & & & \\
\hline & & $35-39(\mathrm{~F})$ & 2163 & & & & & & & & $18 \cdot 2$ & $18 \cdot 0$ & & & & & & & & & & \\
\hline & & $40-44(\mathrm{~F})$ & 2139 & & & & & & & & $17 \cdot 6$ & $15 \cdot 0$ & & & & & & & & & & \\
\hline & & $45-49(F)$ & 1810 & & & & & & & & $18 \cdot 3$ & $16 \cdot 0$ & & & & & & & & & & \\
\hline & & $50-59(\mathrm{~F})$ & 2284 & & & & & & & & $17 \cdot 8$ & $16 \cdot 0$ & & & & & & & & & & \\
\hline & & $60-69(\mathrm{~F})$ & 1339 & & & & & & & & $17 \cdot 1$ & $15 \cdot 0$ & & & & & & & & & & \\
\hline \multirow[t]{6}{*}{ Ireland ${ }^{(89,90)}$} & $2008-2010$ & 18-35 (M) & 276 & 4-d record & 10.7 & 2.9 & $105 \cdot 6$ & 41.6 & $16 \cdot 0$ & 4.9 & 53.9 & $32 \cdot 0$ & 8.1 & $4 \cdot 3$ & & & & & $60 \cdot 7$ & $34 \cdot 3$ & 9.2 & 4.7 \\
\hline & & 36-50 (M) & 205 & & 9.7 & 2.5 & 97.1 & 39.1 & $15 \cdot 8$ & 4.8 & $46 \cdot 4$ & $28 \cdot 8$ & 7.5 & $4 \cdot 1$ & & & & & $50 \cdot 3$ & $30 \cdot 2$ & 8.2 & $4 \cdot 3$ \\
\hline & & 51-64 (M) & 153 & & 9.3 & $2 \cdot 4$ & 96.5 & 39.1 & $16 \cdot 2$ & 4.9 & $43 \cdot 1$ & 27.5 & $7 \cdot 1$ & 4.0 & & & & & 47.6 & 29.1 & 7.9 & $4 \cdot 2$ \\
\hline & & $18-35(F)$ & 255 & & 7.5 & $2 \cdot 3$ & 83.8 & 35.5 & $17 \cdot 8$ & $5 \cdot 1$ & $45 \cdot 6$ & $28 \cdot 2$ & $9 \cdot 2$ & 4.7 & & & & & $50 \cdot 3$ & 29.9 & $10 \cdot 2$ & 4.9 \\
\hline & & $36-50(\mathrm{~F})$ & 232 & & $7 \cdot 1$ & 1.9 & 78.8 & 34.4 & $17 \cdot 1$ & $5 \cdot 0$ & $36 \cdot 4$ & 24.4 & 7.5 & $4 \cdot 1$ & & & & & $40 \cdot 2$ & $26 \cdot 0$ & 8.3 & 4.3 \\
\hline & & $51-64(\mathrm{~F})$ & 153 & & 7.0 & 1.7 & 84.4 & 35.8 & $18 \cdot 7$ & $5 \cdot 2$ & 45.4 & 24.0 & 7.4 & 4.1 & & & & & 38.8 & $25 \cdot 4$ & 8.1 & 4.3 \\
\hline \multirow[t]{6}{*}{ |srael|(91) } & 2014-2016 & 18-34 (M) & & 24-h recall & 9.0 & 4.4 & 87.0 & 58.0 & $16 \cdot 5$ & $9 \cdot 0$ & & & & & & & & & & & & \\
\hline & & 35-44 (M) & & & 7.9 & 3.3 & 79.0 & $49 \cdot 0$ & $16 \cdot 9$ & 8.5 & & & & & & & & & & & & \\
\hline & & 45-64 (M) & & & $7 \cdot 1$ & 3.8 & 69.0 & $56 \cdot 0$ & $16 \cdot 6$ & 9.6 & & & & & & & & & & & & \\
\hline & & $18-34(F)$ & & & $6 \cdot 2$ & 3.0 & 69.0 & 45.0 & $19 \cdot 1$ & $9 \cdot 7$ & & & & & & & & & & & & \\
\hline & & $35-44(F)$ & & & $6 \cdot 2$ & $2 \cdot 7$ & $62 \cdot 0$ & 39.0 & $17 \cdot 3$ & 8.7 & & & & & & & & & & & & \\
\hline & & $45-64(F)$ & & & 5.3 & 2.5 & 64.0 & $45 \cdot 0$ & $21 \cdot 0$ & 11.3 & & & & & & & & & & & & \\
\hline Italy ${ }^{(47)}$ & 2005-2006 & 18-65 (M) & 1068 & 3-d record & $10 \cdot 0$ & $2 \cdot 7$ & 86.0 & 38.0 & 13.5 & 4.7 & & & & & & & & & & & & \\
\hline & & $18-65(F)$ & 1244 & & $8 \cdot 1$ & $2 \cdot 2$ & 79.5 & 33.0 & $15 \cdot 4$ & $5 \cdot 1$ & & & & & & & & & & & & \\
\hline Lithuania $^{(92)}$ & 2013-2014 & 19-34 & 772 & 24-h recall & 8.1 & & 58.1 & & $12 \cdot 8$ & & & & & & & & & & & & & \\
\hline & & $35-49$ & 692 & & 7.8 & & 55.5 & & $12 \cdot 7$ & & & & & & & & & & & & & \\
\hline & & $50-64$ & 749 & & $7 \cdot 4$ & & 54.8 & & $13 \cdot 2$ & & & & & & & & & & & & & \\
\hline $\operatorname{Mexico}^{(48)}$ & 2012 & $20+(M)$ & 3174 & 24-h recall & 9.4 & & & & & & & & $12 \cdot 8$ & & & & & & & & & \\
\hline & & $20+(F)$ & & & $7 \cdot 2$ & & & & & & & & $12 \cdot 2$ & & & & & & & & & \\
\hline Netherlands ${ }^{(49)}$ & 2012-2016 & $19-30(\mathrm{M})$ & 260 & 24-h recall & $11 \cdot 1$ & & 135.0 & & $25 \cdot 8$ & & 81.4 & & 13.0 & & & & & & $90 \cdot 7$ & & 14.6 & \\
\hline & & $31-50$ (M) & 259 & & 11.4 & & 123.0 & & $17 \cdot 7$ & & $67 \cdot 8$ & & $10 \cdot 6$ & & & & & & 75.5 & & $12 \cdot 0$ & \\
\hline & & $51-70(\mathrm{M})$ & 264 & & $10 \cdot 3$ & & 109.0 & & $17 \cdot 4$ & & 55.5 & & 8.9 & & & & & & $62 \cdot 8$ & & $10 \cdot 1$ & \\
\hline
\end{tabular}


Table 6. (Continued)

\begin{tabular}{|c|c|c|c|c|c|c|c|c|c|c|c|c|c|c|c|c|c|c|c|c|c|c|}
\hline \multirow[b]{2}{*}{ Country } & \multirow[b]{2}{*}{ Assessment year } & \multirow[b]{2}{*}{ Age range (years) } & \multirow[b]{2}{*}{$n$} & \multirow[b]{2}{*}{ Method } & \multicolumn{2}{|c|}{ Energy } & \multicolumn{4}{|c|}{ Total sugars } & \multicolumn{4}{|c|}{ Added sugars } & \multicolumn{4}{|c|}{ Sucrose } & \multicolumn{4}{|c|}{ Free sugars } \\
\hline & & & & & MJ & SD & g & SD & $\% \mathrm{E}$ & SD & g & SD & $\% \mathrm{E}$ & SD & g & SD & $\% \mathrm{E}$ & SD & $\mathrm{g}$ & SD & $\% \mathrm{E}$ & $\mathrm{SD}$ \\
\hline & & $19-30(\mathrm{~F})$ & 256 & & 8.0 & & 106.0 & & $26 \cdot 0$ & & 59.8 & & $12 \cdot 3$ & & & & & & 67.6 & & 13.9 & \\
\hline & & $31-50(\mathrm{~F})$ & 264 & & 7.9 & & 98.0 & & $20 \cdot 1$ & & 50.5 & & $10 \cdot 4$ & & & & & & $57 \cdot 0$ & & 11.8 & \\
\hline & & $51-70(\mathrm{~F})$ & 258 & & 7.6 & & 92.0 & & 19.8 & & $42 \cdot 7$ & & $9 \cdot 1$ & & & & & & $48 \cdot 7$ & & $10 \cdot 4$ & \\
\hline New Zealand $\$(80,81)$ & 2008-2009 & $19-30(\mathrm{M})$ & 284 & 24-h recall & 11.9 & & 147.0 & & $20 \cdot 1$ & & 69.7 & & $10 \cdot 8$ & & $73 \cdot 2$ & & $9 \cdot 4$ & & $78 \cdot 1$ & & $12 \cdot 1$ & \\
\hline $31-50(\mathrm{M})$ & 598 & & 11.5 & & 133.0 & & 19.5 & & $62 \cdot 1$ & & 9.5 & & 64.5 & & 8.9 & & $70 \cdot 3$ & & 10.9 & & & \\
\hline 51-70 (M) & 378 & & $9 \cdot 4$ & & 108.0 & & 19.5 & & $46 \cdot 0$ & & 8.4 & & 49.0 & & 8.4 & & 54.4 & & 10.1 & & & \\
\hline $19-30(\mathrm{~F})$ & 434 & & 8.4 & & $123 \cdot 0$ & & 23.8 & & 55.6 & & $12 \cdot 2$ & & $65 \cdot 8$ & & $11 \cdot 1$ & & 62.4 & & $14 \cdot 1$ & & & \\
\hline \multirow[t]{2}{*}{$31-50(F)$} & 746 & & 7.9 & & 98.0 & & 21.3 & & $40 \cdot 1$ & & $9 \cdot 1$ & & $45 \cdot 3$ & & $9 \cdot 6$ & & 47.9 & & 10.8 & & & \\
\hline & & $51-70(\mathrm{~F})$ & 517 & & $7 \cdot 2$ & & 95.0 & & 21.7 & & $35 \cdot 7$ & & 8.6 & & $40 \cdot 4$ & & $9 \cdot 3$ & & 43.2 & & 9.7 & \\
\hline \multirow[t]{8}{*}{ Norway ${ }^{(93)}$} & 2010 & $18-29(\mathrm{M})$ & 138 & 24-h recall & $12 \cdot 8$ & 4.0 & & & & & $69 \cdot 0$ & $55 \cdot 0$ & 9.2 & 6.9 & & & & & & & & \\
\hline & & $30-39(\mathrm{M})$ & 136 & & 11.5 & 3.5 & & & & & 49.0 & 41.0 & $7 \cdot 2$ & 6.0 & & & & & & & & \\
\hline & & 40-49 (M) & 179 & & $10 \cdot 6$ & 3.1 & & & & & $51 \cdot 0$ & $45 \cdot 0$ & $7 \cdot 7$ & 6.0 & & & & & & & & \\
\hline & & 50-59 (M) & 192 & & $10 \cdot 4$ & 3.1 & & & & & $41 \cdot 0$ & 38.0 & $6 \cdot 3$ & $5 \cdot 1$ & & & & & & & & \\
\hline & & $18-29(\mathrm{~F})$ & 143 & & $8 \cdot 1$ & 2.5 & & & & & $46 \cdot 0$ & 41.0 & $9 \cdot 3$ & 6.5 & & & & & & & & \\
\hline & & $30-39(F)$ & 169 & & 8.4 & $2 \cdot 4$ & & & & & $42 \cdot 0$ & 34.0 & $8 \cdot 2$ & $5 \cdot 6$ & & & & & & & & \\
\hline & & $40-49(\mathrm{~F})$ & 256 & & $8 \cdot 1$ & $2 \cdot 4$ & & & & & $32 \cdot 0$ & 24.0 & $6 \cdot 6$ & 4.4 & & & & & & & & \\
\hline & & $50-59(\mathrm{~F})$ & 193 & & 7.9 & 2.3 & & & & & 33.0 & $27 \cdot 0$ & 6.8 & 4.9 & & & & & & & & \\
\hline \multirow[t]{2}{*}{ Portugal ${ }^{(70)}$} & $2015-2016$ & 18-64 (M) & 3056 & 24-h recall & 9.8 & & $92 \cdot 3$ & & $16 \cdot 2$ & & 36.6 & & 6.5 & & & & & & $40 \cdot 0$ & & 6.9 & \\
\hline & & $18-64(F)$ & 3556 & & 6.9 & & 78.0 & & 19.4 & & $30 \cdot 0$ & & $7 \cdot 1$ & & & & & & 32.6 & & 7.9 & \\
\hline Spain $(71,72)$ & 2013 & 18-64 (M) & 798 & 3-d record & 8.2 & $2 \cdot 3$ & 78.4 & $36 \cdot 7$ & $16 \cdot 0$ & $5 \cdot 8$ & & & & & & & & & 35.2 & 27.1 & $7 \cdot 0$ & 4.8 \\
\hline $18-64(F)$ & 857 & & $7 \cdot 0$ & 1.8 & 71.7 & 30.5 & $17 \cdot 3$ & $5 \cdot 7$ & & & & & & & & & 31.6 & $22 \cdot 3$ & 7.3 & 4.5 & & \\
\hline \multirow[t]{5}{*}{ Switzerland ${ }^{(94)}$} & 2014-2015 & $18-75(\mathrm{M})$ & 933 & 24-h recall & & & 114.0 & $49 \cdot 0$ & $18 \cdot 1$ & & $61 \cdot 0$ & $41 \cdot 0$ & 9.4 & & & & & & 73.0 & 44.0 & $11 \cdot 4$ & \\
\hline & & $18-75(\mathrm{~F})$ & 1124 & & & & 99.0 & 37.0 & 20.5 & & $46 \cdot 0$ & 28.0 & 9.3 & & & & & & 57.0 & 32.0 & 11.5 & \\
\hline & & $18-29$ & 400 & & & & 113.0 & $47 \cdot 0$ & $19 \cdot 6$ & & 63.0 & 41.0 & $10 \cdot 5$ & & & & & & $75 \cdot 0$ & $45 \cdot 0$ & $12 \cdot 7$ & \\
\hline & & $30-64$ & 1319 & & & & 107.0 & 44.0 & $19 \cdot 2$ & & 53.0 & 36.0 & 9.3 & & & & & & $65 \cdot 0$ & 39.0 & $11 \cdot 4$ & \\
\hline & & $65-75$ & 338 & & & & 99.0 & 38.0 & 19.5 & & $42 \cdot 0$ & $25 \cdot 0$ & 8.1 & & & & & & 53.0 & 28.0 & $10 \cdot 2$ & \\
\hline \multirow[t]{2}{*}{$\mathrm{UK}^{(73)}$} & $2014 / 15-2015 / 16$ & 19-64 (M) & 450 & 4-d record & $8 \cdot 3$ & 2.4 & & & & & & & & & & & & & $64 \cdot 3$ & 46.6 & $11 \cdot 1$ & 6.4 \\
\hline & & $19-64(\mathrm{~F})$ & 632 & 4-d record & $6 \cdot 6$ & $2 \cdot 0$ & & & & & & & & & & & & & $50 \cdot 0$ & 34.4 & $11 \cdot 2$ & $6 \cdot 1$ \\
\hline \multirow[t]{9}{*}{ United States**(53,55) } & $2017-2018$ & $20-29(\mathrm{M})$ & 335 & 24-h recall & $10 \cdot 1$ & 0.3 & $105 \cdot 0$ & 3.6 & & & & & & & & & & & & & & \\
\hline & & 30-39 (M) & 329 & 24-h recall & 11.1 & 0.3 & 124.0 & $5 \cdot 8$ & & & & & & & & & & & & & & \\
\hline & & 40-49 (M) & 340 & 24-h recall & $10 \cdot 9$ & 0.5 & 128.0 & 9.5 & & & & & & & & & & & & & & \\
\hline & & 50-59 (M) & 375 & 24-h recall & $10 \cdot 7$ & 0.2 & 128.0 & 7.1 & & & & & & & & & & & & & & \\
\hline & & $20-29(\mathrm{~F})$ & 359 & 24-h recall & 8.3 & 0.2 & 99.0 & 4.8 & & & & & & & & & & & & & & \\
\hline & & $30-39(\mathrm{~F})$ & 407 & 24-h recall & 7.9 & 0.2 & 96.0 & 4.0 & & & & & & & & & & & & & & \\
\hline & & $40-49(\mathrm{~F})$ & 367 & 24-h recall & 8.0 & 0.3 & 106.0 & $5 \cdot 8$ & & & & & & & & & & & & & & \\
\hline & & $50-59(\mathrm{~F})$ & 419 & 24-h recall & 7.6 & 0.4 & 97.0 & 8.2 & & & & & & & & & & & & & & \\
\hline & 2009-2012 & $19+$ & 10697 & 24-h recall & & & & & & & $62 \cdot 1$ & & $13 \cdot 1$ & & & & & & & & & \\
\hline
\end{tabular}

M: male; F: female.

† Total sugars for Andorra reported as digestible sugars.

₹ Canada variance reported as standard error (SE), not SD. Intakes for adults not split by sex, based on the first day recall, not usual intake.

$\$$ Chile, Germany and New Zealand report median values.

Greece $n$-values include older adults, but data are for age groups as reported.

"Hungary mono- and disaccharides added to foods, foodstuffs during production or preparation, and natural sugars in honey.

** United States reports SEM. 
Table 7. Energy and sugar intake of older adults (65+ years)

\begin{tabular}{|c|c|c|c|c|c|c|c|c|c|c|c|c|c|c|c|c|c|c|c|c|c|c|}
\hline \multirow[b]{2}{*}{ Country } & \multirow[b]{2}{*}{ Assessment year } & \multirow[b]{2}{*}{ Age range (years) } & \multirow[b]{2}{*}{$n$} & \multirow[b]{2}{*}{ Method } & \multicolumn{2}{|c|}{ Energy } & \multicolumn{4}{|c|}{ Total sugars } & \multicolumn{4}{|c|}{ Added sugars } & \multicolumn{4}{|c|}{ Sucrose } & \multicolumn{4}{|c|}{ Free sugars } \\
\hline & & & & & MJ & SD & $g$ & SD & $\% \mathrm{E}$ & SD & $g$ & SD & $\% \mathrm{E}$ & SD & $g$ & SD & $\% \mathrm{E}$ & SD & $g$ & SD & $\% \mathrm{E}$ & SD \\
\hline \multirow[t]{2}{*}{ Andorra $^{\dagger}(74)$} & 2004-2005 & $65-75(\mathrm{M})$ & 45 & 24-h recall & 7.0 & 0.9 & 79.6 & 14.6 & $19 \cdot 1$ & $2 \cdot 8$ & & & & & & & & & & & & \\
\hline & & $65-75(\mathrm{~F})$ & 48 & & 6.4 & 1.0 & 82.5 & 19.1 & $22 \cdot 2$ & 3.9 & & & & & & & & & & & & \\
\hline \multirow[t]{2}{*}{ Australia $(35,36)$} & 2011-2012 & $71+(M)$ & & 24-h recall & 8.2 & & 101.8 & & 19.8 & & $45 \cdot 3$ & & 8.5 & & & & & & 52.9 & & $10 \cdot 0$ & \\
\hline & & $71+(F)$ & & & 6.6 & & 84.4 & & $20 \cdot 3$ & & 34.2 & & 8.0 & & & & & & 40.7 & & 9.5 & \\
\hline \multirow{2}{*}{ Austria( $^{(83)}$} & 2012 & $65-80(\mathrm{M})$ & 76 & 3-d record & 8.0 & 0.0 & & & & & & & & & & & 8.0 & & & & & \\
\hline & & $65-80(\mathrm{~F})$ & 100 & & 7.0 & & & & & & & & & & & & 9.0 & & & & & \\
\hline Brazil| ${ }^{(57)}$ & $2008-2009$ & $60+(M)$ & 1993 & 24-h recall & $7 \cdot 7$ & 2.5 & & & & & $52 \cdot 0$ & 34.0 & 11.0 & $6 \cdot 0$ & & & & & & & & \\
\hline & & $60+(F)$ & 2328 & & 6.5 & $2 \cdot 1$ & & & & & 46.0 & 30.0 & $12 \cdot 0$ & $6 \cdot 0$ & & & & & & & & \\
\hline Chile $^{\ddagger}(38)$ & 2010 & $65+$ & 906 & 24-h recall & & & $42 \cdot 9$ & & & & & & & & & & & & & & & \\
\hline & & $65+(M)$ & & & 7.1 & & & & & & & & & & & & & & & & & \\
\hline & & $65+(F)$ & & & 5.5 & & & & & & & & & & & & & & & & & \\
\hline Denmark $^{(60)}$ & $2011-2013$ & $65-75(\mathrm{M})$ & 262 & 7-d record & $10 \cdot 8$ & 3.1 & & & & & 53.0 & 41.3 & 8.0 & 4.9 & & & & & & & & \\
\hline & & $65-75(\mathrm{~F})$ & 262 & & 8.3 & $2 \cdot 2$ & & & & & 38.0 & 25.9 & 8.0 & 4.1 & & & & & & & & \\
\hline Estonia $^{(40)}$ & 2015 & 60-64 (M) & 85 & 24-h recall & 8.1 & & & & & & & & & & $40 \cdot 0$ & & & & & & & \\
\hline & & 65-69 (M) & 83 & & $7 \cdot 8$ & & & & & & & & & & 34.0 & & & & & & & \\
\hline & & $70-74$ (M) & 74 & & 7.6 & & & & & & & & & & 36.0 & & & & & & & \\
\hline & & $60-64(\mathrm{~F})$ & 187 & & $6 \cdot 2$ & & & & & & & & & & 39.0 & & & & & & & \\
\hline & & $65-69(\mathrm{~F})$ & 194 & & $6 \cdot 3$ & & & & & & & & & & 36.0 & & & & & & & \\
\hline & & $70-74(\mathrm{~F})$ & 147 & & 5.6 & & & & & & & & & & $32 \cdot 0$ & & & & & & & \\
\hline Finland ${ }^{(84)}$ & 2017 & 65-74 (M) & 204 & 24-h recall & 8.0 & & & & & & & & & & 38.0 & & 8.2 & & & & & \\
\hline & & $65-74(F)$ & 247 & & 6.6 & & & & & & & & & & 34.0 & & 8.8 & & & & & \\
\hline France $^{(42)}$ & 2014-2015 & 65-79 (M) & 724 & 24-h recall & 9.3 & 3.4 & 89.4 & $47 \cdot 7$ & & & & & & & & & & & & & & \\
\hline & & $65-79(\mathrm{~F})$ & & & 6.9 & $2 \cdot 7$ & 77.6 & 38.5 & & & & & & & & & & & & & & \\
\hline Germany(95) & 2005-2006 & $65-80(\mathrm{M})$ & 1165 & 24-h recall & 8.9 & & & & & & & & & & & & $9 \cdot 1$ & & & & & \\
\hline & & $65-80(\mathrm{~F})$ & 1331 & & 6.8 & & & & & & & & & & & & 11.3 & & & & & \\
\hline Greece ${ }^{\S(86)}$ & $1995-2000$ & 65-74 (M) & & 24-h recall & & & $92 \cdot 1$ & & & & & & & & & & & & & & & \\
\hline & & $65-74(F)$ & & & & & 86.7 & & & & & & & & & & & & & & & \\
\hline Hungary(87) & 2014 & $\geq 65(\mathrm{M})$ & 372 & 3-d record & 8.1 & & & & & & & & 6.0 & & & & & & & & & \\
\hline & & $\geq 65(F)$ & 485 & & 10.0 & & & & & & & & $7 \cdot 4$ & & & & & & & & & \\
\hline Iceland( 88$)$ & $2010-2011$ & $61-80(\mathrm{M})$ & 151 & 24-h recall & 8.7 & $2 \cdot 8$ & 80.0 & 44.0 & & & 35.0 & 31.0 & & & & & & & & & & \\
\hline & & $61-80(\mathrm{~F})$ & 167 & & 6.7 & 2.0 & 74.0 & $32 \cdot 0$ & & & 51.0 & 24.0 & & & & & & & & & & \\
\hline India (rural) $)^{(64)}$ & $2011-2012$ & $70-79$ (M) & 560 & 24-h recall & & & & & & & 14.0 & 14.0 & & & & & & & & & & \\
\hline & & $80+(M)$ & 180 & & & & & & & & $12 \cdot 4$ & 11.0 & & & & & & & & & & \\
\hline & & $70-79(\mathrm{~F})$ & 571 & & & & & & & & 12.5 & $16 \cdot 0$ & & & & & & & & & & \\
\hline & & $80+(F)$ & 192 & & & & & & & & 11.9 & $10 \cdot 0$ & & & & & & & & & & \\
\hline India (urban) ${ }^{(64)}$ & $2015-2016$ & $70-79(\mathrm{M})$ & 486 & 24-h recall & & & & & & & $17 \cdot 2$ & $16 \cdot 0$ & & & & & & & & & & \\
\hline & & $80+(\mathrm{M})$ & 143 & & & & & & & & $15 \cdot 1$ & $13 \cdot 0$ & & & & & & & & & & \\
\hline & & $70-79(\mathrm{~F})$ & 561 & & & & & & & & 16.9 & $16 \cdot 0$ & & & & & & & & & & \\
\hline & & $80+(F)$ & 207 & & & & & & & & 16.5 & $12 \cdot 0$ & & & & & & & & & & \\
\hline Ireland $(89,90)$ & $2008-2010$ & $65+(M)$ & 106 & 4-d record & 8.3 & 2.6 & 89.5 & 37.0 & $16 \cdot 8$ & 4.9 & 39.5 & $25 \cdot 7$ & $7 \cdot 2$ & 4.0 & & & & & $43 \cdot 1$ & $27 \cdot 1$ & $7 \cdot 8$ & 4.2 \\
\hline & & $65+(F)$ & 120 & & 6.5 & 1.6 & 83.4 & $35 \cdot 8$ & $19 \cdot 6$ & 5.4 & 35.3 & $24 \cdot 1$ & $7 \cdot 7$ & 4.3 & & & & & 39.5 & $25 \cdot 8$ & $8 \cdot 8$ & 4.5 \\
\hline Israel|(91) & 2014-2015 & 65-74 (M) & & 24-h recall & $6 \cdot 7$ & $2 \cdot 9$ & $67 \cdot 0$ & 48.0 & $16 \cdot 1$ & 8.9 & & & & & & & & & & & & \\
\hline & & 75-84 (M) & & & 5.9 & 2.5 & $56 \cdot 0$ & 37.0 & $15 \cdot 7$ & 8.8 & & & & & & & & & & & & \\
\hline & & $85+(\mathrm{M})$ & & & $5 \cdot 2$ & $2 \cdot 1$ & 59.0 & $40 \cdot 0$ & 18.5 & $10 \cdot 6$ & & & & & & & & & & & & \\
\hline & & $65-74(\mathrm{~F})$ & & & 5.5 & 2.4 & 62.0 & 38.0 & $17 \cdot 8$ & 7.8 & & & & & & & & & & & & \\
\hline & & $75-84(\mathrm{~F})$ & & & 4.8 & 1.9 & 50.0 & $30 \cdot 0$ & 16.9 & 7.8 & & & & & & & & & & & & \\
\hline & & $85+(F)$ & & & 4.6 & 1.7 & 55.0 & 37.0 & $18 \cdot 6$ & $9 \cdot 8$ & & & & & & & & & & & & \\
\hline
\end{tabular}


Nutrition Research Reviews

Table 7. (Continued)

\begin{tabular}{|c|c|c|c|c|c|c|c|c|c|c|c|c|c|c|c|c|c|c|c|c|c|c|}
\hline \multirow[b]{2}{*}{ Country } & \multirow[b]{2}{*}{ Assessment year } & \multirow[b]{2}{*}{ Age range (years) } & \multirow[b]{2}{*}{$n$} & \multirow[b]{2}{*}{ Method } & \multicolumn{2}{|c|}{ Energy } & \multicolumn{4}{|c|}{ Total sugars } & \multicolumn{4}{|c|}{ Added sugars } & \multicolumn{4}{|c|}{ Sucrose } & \multicolumn{4}{|c|}{ Free sugars } \\
\hline & & & & & MJ & SD & $\mathrm{g}$ & SD & $\% \mathrm{E}$ & SD & g & SD & $\% \mathrm{E}$ & SD & $\mathrm{g}$ & SD & $\% \mathrm{E}$ & SD & g & SD & $\% \mathrm{E}$ & SD \\
\hline Italy ${ }^{(47)}$ & 2005-2006 & $65+(\mathrm{M})$ & 202 & 3-d record & 9.6 & $2 \cdot 3$ & $81 \cdot 6$ & $35 \cdot 0$ & $13 \cdot 3$ & $5 \cdot 0$ & & & & & & & & & & & & \\
\hline Lithuania $^{(92)}$ & 2013-2014 & $\begin{array}{c}65+(F) \\
65-75\end{array}$ & 316 & 24-h recall & $\begin{array}{l}7.7 \\
6.7\end{array}$ & $2 \cdot 0$ & 78.6 & $32 \cdot 0$ & $16 \cdot 2$ & $5 \cdot 4$ & & & & & & & & & & & & \\
\hline Mexico $\neq^{(96)}$ & 2012 & $\begin{array}{l}65+(\mathrm{M}) \\
65+(\mathrm{F})\end{array}$ & $\begin{array}{l}237 \\
289\end{array}$ & SFFQ & $\begin{array}{c}0 \cdot 1 \\
7 \cdot 1 \\
5 \cdot 5^{\ddagger}\end{array}$ & & $\begin{array}{r}01.1 \\
87.8 \\
71.5^{\ddagger}\end{array}$ & & 13.2 & & & & & & & & & & & & & \\
\hline Netherlands ${ }^{(49)}$ & 2012-2016 & $\begin{array}{l}71-79(\mathrm{M}) \\
71-79(\mathrm{~F})\end{array}$ & $\begin{array}{l}260 \\
257\end{array}$ & 24-h recall & $\begin{array}{l}9 \cdot 1 \\
7 \cdot 4\end{array}$ & & $\begin{array}{l}99.0 \\
88.0\end{array}$ & & $\begin{array}{l}19 \cdot 0 \\
20 \cdot 6\end{array}$ & & $\begin{array}{l}49 \cdot 1 \\
38 \cdot 9\end{array}$ & & $\begin{array}{l}8.0 \\
8.5\end{array}$ & & & & & & $\begin{array}{l}55 \cdot 4 \\
44.2\end{array}$ & & $\begin{array}{l}9.0 \\
9.8\end{array}$ & \\
\hline New Zealand ${ }^{\ddagger}(81)$ & 2008-2009 & $\begin{array}{l}71+(\mathrm{M}) \\
71+(\mathrm{F})\end{array}$ & $\begin{array}{l}480 \\
585\end{array}$ & 24-h recall & $\begin{array}{l}8 \cdot 1 \\
6 \cdot 1\end{array}$ & & $\begin{array}{r}105.0 \\
84.0\end{array}$ & & $\begin{array}{l}21 \cdot 1 \\
23 \cdot 3\end{array}$ & & $\begin{array}{l}45 \cdot 9 \\
32 \cdot 2\end{array}$ & & $\begin{array}{l}8 \cdot 8 \\
8.4\end{array}$ & & $\begin{array}{l}45.9 \\
33.5\end{array}$ & & $\begin{array}{l}9 \cdot 1 \\
9.7\end{array}$ & & $\begin{array}{l}51.9 \\
36.8\end{array}$ & & $\begin{array}{r}10 \cdot 1 \\
9.9\end{array}$ & \\
\hline Norway ${ }^{(93)}$ & 2010 & $\begin{array}{l}60-70(\mathrm{M}) \\
60-70(\mathrm{~F})\end{array}$ & $\begin{array}{l}217 \\
164\end{array}$ & 24-h recall & $\begin{array}{l}9.9 \\
7.4\end{array}$ & $\begin{array}{l}2 \cdot 9 \\
2 \cdot 2\end{array}$ & & & & & $\begin{array}{l}39.0 \\
30.0\end{array}$ & $\begin{array}{l}32 \cdot 0 \\
24 \cdot 0\end{array}$ & $\begin{array}{l}6 \cdot 3 \\
6 \cdot 5\end{array}$ & $\begin{array}{l}4 \cdot 4 \\
4.2\end{array}$ & & & & & & & & \\
\hline Portugal ${ }^{(70)}$ & 2015-2016 & $\begin{array}{l}65-84(\mathrm{M}) \\
65-84(\mathrm{~F})\end{array}$ & $\begin{array}{l}857 \\
790\end{array}$ & 24-h recall & $\begin{array}{l}8.3 \\
6 \cdot 3\end{array}$ & & $\begin{array}{l}72 \cdot 3 \\
71.9\end{array}$ & & $\begin{array}{l}15.6 \\
19.9\end{array}$ & & $\begin{array}{l}20 \cdot 9 \\
19 \cdot 1\end{array}$ & & $\begin{array}{l}4 \cdot 0 \\
5 \cdot 2\end{array}$ & & & & & & $\begin{array}{l}22 \cdot 6 \\
23 \cdot 3\end{array}$ & & $\begin{array}{l}4 \cdot 4 \\
6 \cdot 2\end{array}$ & \\
\hline Spain ${ }^{(71,72)}$ & 2013 & $\begin{array}{l}65-75(\mathrm{M}) \\
65-75(\mathrm{~F})\end{array}$ & $\begin{array}{r}99 \\
107\end{array}$ & 3-d record & $\begin{array}{l}7.4 \\
6.2\end{array}$ & $\begin{array}{l}2.0 \\
1.5\end{array}$ & $\begin{array}{l}74 \cdot 2 \\
71 \cdot 8\end{array}$ & $\begin{array}{l}37.4 \\
30.6\end{array}$ & $\begin{array}{l}16 \cdot 7 \\
19.8\end{array}$ & $\begin{array}{l}6 \cdot 1 \\
6 \cdot 9\end{array}$ & & & & & & & & & $\begin{array}{l}21 \cdot 3 \\
20 \cdot 1\end{array}$ & $\begin{array}{l}15 \cdot 2 \\
14.7\end{array}$ & $\begin{array}{l}4.8 \\
5.4\end{array}$ & $\begin{array}{l}3.1 \\
3.7\end{array}$ \\
\hline $\mathrm{UK}^{(73)}$ & $2014 / 15-2015 / 16$ & $\begin{array}{c}65-74(\mathrm{M}) \\
75+(\mathrm{M})\end{array}$ & $\begin{array}{l}71 \\
70\end{array}$ & 4-d record & $\begin{array}{l}7.7 \\
7.4\end{array}$ & $\begin{array}{l}1 \cdot 7 \\
2.0\end{array}$ & & & & & & & & & & & & & $\begin{array}{l}62.0 \\
60.5\end{array}$ & $\begin{array}{l}34.3 \\
35.7\end{array}$ & $\begin{array}{l}11.8 \\
12.5\end{array}$ & $\begin{array}{l}5 \cdot 8 \\
6 \cdot 4\end{array}$ \\
\hline & & $\begin{array}{c}65-74(F) \\
75+(F)\end{array}$ & $\begin{array}{r}110 \\
84\end{array}$ & & $\begin{array}{l}6.1 \\
5.6\end{array}$ & $\begin{array}{r}1.8 \\
1.5\end{array}$ & & & & & & & & & & & & & $\begin{array}{l}42 \cdot 1 \\
37.7\end{array}$ & $\begin{array}{l}30 \cdot 0 \\
20 \cdot 1\end{array}$ & $\begin{array}{l}10 \cdot 4 \\
10.4\end{array}$ & $\begin{array}{l}7 \cdot 1 \\
4.9\end{array}$ \\
\hline United States ${ }^{\|(55)}$ & 2017-2018 & 60-69 (M) & 500 & 24-h recall & $\begin{array}{r}10 \cdot 3 \\
9.0\end{array}$ & 0.3 & $\begin{array}{l}116 \cdot 0 \\
111 \cdot 0\end{array}$ & $4 \cdot 3$ & & & & & & & & & & & (7) & & 10.4 & \\
\hline & & $\begin{array}{c}60-69(\mathrm{~F}) \\
70+(\mathrm{F})\end{array}$ & $\begin{array}{l}479 \\
404\end{array}$ & & $\begin{array}{l}7.0 \\
7.0\end{array}$ & $\begin{array}{l}0.2 \\
0.3 \\
0.2\end{array}$ & $\begin{array}{l}90.0 \\
88.0\end{array}$ & $\begin{array}{l}7.0 \\
2.4\end{array}$ & & & & & & & & & & & & & & \\
\hline
\end{tabular}

† Total sugars for Andorra reported as digestible sugars.

$\neq$ Chile, Mexico and New Zealand report median.

$\ddagger$ Chile, Mexico and New Zealand report median.
$\S$-Values combined for $19-59$ and $65-74$ years for Greece.

"United States reports SEM. 
adolescents. The second outcome is that more data (both in terms of quantity and harmonisation) are required to give a complete picture of sugars intake globally. Caution is needed when interpreting these dietary intake data across sugar types and making inter-country comparisons, due to differences in the dietary assessment methodologies, differences in the time periods in which dietary intake was assessed and differences in national clustering of age groups. Future studies could examine the feasibility of identifying and compensating for selected under-reporting (which may be worse for sugar-sweetened foods and beverages) by using biomarkers in a subset of the population.

\section{Interpretation and application of dietary sugar data Challenges of data availability and quality}

Whilst this review serves to update data on sugar intake from two previous reviews, for some countries there have been no updates since the time of the previous reviews, and hence these earlier data are included here also. New data are, however, available and included for countries such as Andorra, Australia, Chile, China, Estonia, Germany, Greece, India, Lithuania, Mexico, New Zealand, Portugal, Slovenia and Switzerland, and updated data are available for some age groups, including for Belgium, Canada, Denmark, Finland, France, Germany, Ireland, Israel, Netherlands, Norway, the UK and the United States.

Notwithstanding the addition of new data, the findings suggest that relatively few countries globally provide any kind of dietary data on sugar intake. The greatest amount of intake data was available for adults, but even then, data were only available for 29 countries; mostly in the European Union (EU) and greater European region, Australia and New Zealand, the United States and some of the Americas. Of note, there are 194 countries in the world today ${ }^{(99)}$ and 100 countries which have food-based dietary guidelines ${ }^{(26)}$. Significant gaps remain in examining sugars intakes globally, mirroring gaps in dietary surveillance in general $^{(100)}$.

A notable difference between this 2020 review and previous reviews ${ }^{(15,16)}$ is the inclusion of available data for dietary free sugar intake. This is in part due to newly available methods and definitions to estimate free sugars in databases of food consumption $^{(101,102)}$. These new data allow comparisons against nutrient goals for dietary sugars, which are typically based on free sugars intake. In particular, the inclusion of these data highlights the challenges of meeting dietary recommendations for free sugars, particularly at 5\%E. For example, of the twentyseven surveys that provided an estimate for free sugars, only one survey reported an estimate of mean intake below that value (Spain, where older male adults reported sugars intakes of $4.8 \%$ E). Understanding which food groups are the key contributors to free sugars at each lifecycle stage will help to inform strategies to reduce these intakes ${ }^{(103)}$.

Nevertheless, there remain considerable challenges in estimating dietary sugar intake data. Standard challenges exist relating to population sampling, selection bias and the assumptions and uncertainties inherent to the dietary collection methods used (24 h recall, food records and food frequency questionnaires $)^{(15,100)}$. Further, for sugars, obtaining reliable estimates of added and free sugar consumption continues to be a challenge for researchers. Despite the development of protocols to estimate added/free sugar composition ${ }^{(101,104)}$, detailed food and composition data at brand level are necessary for these purposes $^{(15,102)}$. As part of EFSA's ongoing assessment to provide scientific advice on an tolerable upper intake level for sugars, harmonised data on dietary sugars intake will be compared for countries in the European region; however, challenges remain for assuring valid comparisons in other global regions ${ }^{(14)}$.

\section{Application of dietary sugar data}

For dietary intake researchers, data managers and risk assessors, the need to survey and accurately quantify dietary sugars intake remains a priority. However, it also remains problematic, given the lack of global harmonisation of definitions, classifications and recommendations and the lack of biomarkers to differentiate between the dietary intake of different sugars types. Future situations that could overcome this lack of harmony, but that could also offer objective biomarkers of intake and quantitative measures of the amounts of the various sugar forms present within foods, would be welcome. However, where available, these are currently only in their infancy and, indeed for biomarkers at least, may be a futile effort ${ }^{(105-108)}$.

For policy makers, current dietary sugar intake patterns are needed for consistent longitudinal monitoring and may enforce concerns and underline the importance of policy initiatives to reduce population sugar and energy (calorie) intakes. To date, there have been a variety of approaches undertaken by different countries, ranging from education strategies, taxation policies on single food types (e.g. taxes on sugar-sweetened beverages) to more comprehensive initiatives affecting multiple food types (e.g. UK initiatives targeting reformulation, portion size and altering consumer purchasing behaviour ${ }^{(109)}$, or indeed a mix of all three $\left.\mathrm{e}^{(110,111)}\right)$. Although there is some information to suggest that such efforts may reduce purchase and, in some cases, intake, any impact on outcomes other than absolute dietary intakes (e.g. health outcomes such as obesity risk) remain unknown, despite numerous modelling exercises ${ }^{(112-117)}$. A challenge remains in balancing the need to have regular robust population dietary assessments to underpin and evaluate policy with the costs and logistics that accompany such surveillance programmes ${ }^{(100)}$.

Food compositional data for sugars are also used on food labels as applied by the food industry. The values on such labels can be used to underpin estimates of dietary sugars intake by researchers. For most countries, there is no mandatory labelling of added or free sugars, and only total sugars is required to be declared on the label, mainly due the current lack of a an accepted analytical method for manufacturers to assess added/free sugar content in foods/drinks. Exceptions include the United States, where added sugars ( $\mathrm{g}$, \% daily value) will be included on the food label from 2021 ${ }^{(118)}$, and Mexico, who have recently (2020) also updated their labelling guidelines to include added sugars ${ }^{(119)}$. Notwithstanding this, challenges remain: for example, in the United States, single-ingredient sugars such as syrups are treated differently with respect to labelling requirements and are not labelled as added sugars unless added 
as an ingredient in other foods. Given such disparities, continued challenges will remain when sourcing and applying food composition data to underpin estimates or modelling of dietary sugars intake, particularly for foods which are either new to market or have been recently reformulated for sugars. It is promising to see a recently proposed method to quantify free sugars in foods and beverages which, if workable, will be a valuable addition for assessment of free sugars and will be useful in product development and for public health bodies, industry and regulatory $\operatorname{agencies}^{(107)}$.

A further challenge relates to consumer awareness and understanding of the meaning of the terms 'added' or 'free' sugars in the context of dietary guidance. As mentioned, globally, food labels mostly only provide the interested consumer with information on quantitative amounts of total sugars present. Recent studies have suggested confusion in recognising dietary sources of added or free sugars, with results reiterating the need to have a readily understood term for sugars relating to label information and to educate and inform consumers of such $^{(120,121)}$. With disconnection between dietary guidance and food labelling regulations with respect to total/free/added sugars, it will be a challenge for consumers to understand the relevant sugar content of foods from the nutrition label.

\section{Future directions and conclusion}

With the role of dietary sugars under scrutiny, this review aimed to provide a current picture of dietary guidance and actual sugar intake worldwide. It shows that, globally, a minority of countries provide any estimate of sugar intake. Further, intakes of the sugar forms upon which the greatest emphasis is placed (free and added sugars) generally exceed existing dietary guidelines across all population groups and are highest in children and adolescents and lowest for older adults Whilst individual studies suggest some reductions in intakes of added sugars (\%E) in children and adolescents, this review suggests that there is a need for more data to understand the complete picture. Furthermore, this review suggests a greater need for harmonisation of terms and for collaborative efforts to ensure that the most up-to-date food composition data are used to underpin any estimates of intake or modelling scenarios. Development and consensus on robust, valid biomarkers which provide accurate measures of dietary sugars intake, and preferably in a manner which is cost-effective, non-invasive and quick, would be welcome. In the interim, continued focus on monitoring of both dietary intakes and the efficacy of any public health initiatives remains paramount.

\section{Acknowledgements}

We wish to thank the authors of the dietary survey reports in different countries who assisted us with data related queries.

\section{Financial support}

This work was supported by the World Sugar Research Organisation (WSRO). WSRO had no role in the analysis or writing of this review.
J.W. received financial support from the WSRO for the initial draft of the manuscript. No funding was received for later drafts or for final manuscript. R.R. and H.B. are previous employees of WSRO and were employees at the time that the data were collected.

R.R. and J.W. conceived the idea of this review paper. H.B. collated the data. J.W. and A.N. interpreted the data and drafted the manuscript. All authors read and approved the final version of the manuscript.

\section{References}

1. Fidler Mis N, Braegger C, Bronsky J, et al. (2017) Sugar in infants, children and adolescents: a position paper of the European Society for Paediatric Gastroenterology, Hepatology a nd Nutrition Committee on Nutrition. J Pediatr Gastroenterol Nutr 65, 681-696.

2. Clemens RA, Jones JM, Kern M, et al. (2016) Functionality of sugars in foods and health. Comprehensive Reviews in Food Science and Food Safety. Blackwell Publishing Inc.; 15, 433-470.

3. European Food Safety Authority (2010) Scientific opinion on dietary reference values for carbohydrates and dietary fibre. EFSA J [Internet] 8, 1462. Available from: http://www.efsa. europa.eu/sites/default/files/scientific_output/files/main_doc uments/1462.pdf.

4. World Health Organization (2015) WHO Guideline: sugars intake for adults and children. Geneva: WHO.

5. Scientific Advisory Committee on Nutrition (2015) Carbohydrates and Health. http://www.gov.uk/government/ uploads/system/uploads/attachment_data/file/445503/SACN_ Carbohydrates_and_Health.pdf (accessed January 2020).

6. Prinz P (2019) The role of dietary sugars in health: molecular composition or just calories? Eur J Clin Nutr 73, 1216-1223.

7. Johnson RK, Appel LJ, Brands M, et al. (2009) Dietary sugars intake and cardiovascular health: a scientific statement from the American Heart Association. Circulation 1011-1120.

8. Tappy L, Morio B, Azzout-Marniche D, et al. (2018) French recommendations for sugar intake in adults: a novel approach chosen by ANSES. Nutrients $\mathbf{1 0}, 989$.

9. Institute of Medicine (2005) Dietary reference intakes for energy, carbohydrate, fiber, fat, fatty acids, cholesterol, protein, and amino acids (macronutrients). Washington, DC: The National Academies Press.

10. Jamnik J, Rehman S, Blanco Mejia S, et al. (2016) Fructose intake and risk of gout and hyperuricemia: a systematic review and meta-analysis of prospective cohort studies. BMJ Open 6, e013191.

11. Dornas WC, de Lima WG, Pedrosa ML, et al. (2015) Health implications of high-fructose intake and current research. Adv Nutr 6, 729-737.

12. Lobstein T (2014) WHO | Reducing consumption of sugarsweetened beverages to reduce the risk of childhood overweight and obesity [Internet]. World Health Organization [cited 2020 Jun 14]. Available from: https://www.who.int/ elena/titles/ssbs_childhood_obesity/en/

13. Te Morenga L, Mallard S \& Mann J (2013) Dietary sugars and body weight: systematic review and meta-analyses of randomised controlled trials and cohort studies. BMJ 346, e7492.

14. European Food Safety Authority (2018) Protocol for the scientific opinion on the Tolerable Upper Intake Level of dietary sugars. EFSA J 16, 5393-5440. 
15. Newens KJ \& Walton J (2016) A review of sugar consumption from nationally representative dietary surveys across the world. J Hum Nutr Diet 29, 225-40.

16. Azaïs-Braesco V, Sluik D, Maillot M, et al. (2017) A review of total \& added sugar intakes and dietary sources in Europe. Nutr J 16, 6 .

17. Fisberg M, Kovalskys I, Gómez G, et al. (2018) Total and added sugar intake: assessment in eight Latin American countries. Nutrients 10, 389.

18. Mela DJ \& Elizabeth MW (2018) Perspective: total, added, or free? What kind of sugars should we be talking about? Adv Nutr 9, 63-69.

19. Buyken AE, Mela DJ, Dussort P, et al. (2018) Dietary carbohydrates: a review of international recommendations and the methods used to derive them. Eur J Clin Nutr 72, $1625-1643$.

20. DRI (2005) Dietary reference intakes for energy, carbohydrate, fiber, fat, fatty acids, cholesterol, protein, and amino acids (macronutrients). Washington DC: The National Acadamies Press.

21. European Food Safety Authority (2018) Protocol for the scientific opinion on the Tolerable Upper Intake Level of dietary sugars. EFSA J 16, 5393

22. WHO/FAO (1998) Carbohydrates in human nutrition. Report of a Joint FAO/WHO Expert Consultation. FAO Food Nutr. Pap 66, 1-140.

23. U.S. Department of Agriculture and U.S. Department of Health and Human Services (2020) Dietary Guidelines for Americans, 2020-2025, 9th Edn. [Internet]. Available from: DietaryGuidelines.gov.

24. Institute of Medicine (2005) Dietary reference intakes for energy, carbohydrate, fiber, fat, fatty acids, cholesterol, protein, and amino acids. Washington, DC: National Academies Press, 1-1331.

25. Nordic Council of Ministers (2012) Nordic nutrition recommendations 2012: integrating nutrition and physical activity. Copenhagen: Nordic Council of Ministers, Narayna Press

26. FAO (2003) Food based dietary guidelines in the WHO European Region Nutrition and Food Security Programme. WHO Regional Office for Europe [Internet]. Available from: http://www.euro.who.int.

27. Committee on Medical Aspects of Food Policy (COMA) (1991) Dietary reference values for food energy and nutrients for the United Kingdom. London: HMSO.

28. Ernst JB, Arens-Azevêdo U, Bitzer B, et al. (2019) Quantitative Empfehlung zur Zuckerzufuhr in Deutschland Kurzfassung des Konsensuspapiers der Deutschen AdipositasGesellschaft e. V. (DAG), der Deutschen Diabetes Gesellschaft e. V. (DDG) und der Deutschen Gesellschaft für Ernährung e. V. (DGE). Ernahrungs Umschau [Internet], [cited 2020 Jun 16] 66, 26-34. Available from: www.dge.de/ fileadmin/public/

29. Vos MB, Kaar JL, Welsh JA, et al. (2017) Added sugars and cardiovascular disease risk in children: a scientific statement from the American Heart Association. Circulation 135, e1017-e1034.

30. Erickson J, Sadeghirad B, Lytvyn L, et al. (2017) The scientific basis of guideline recommendations on sugar intake: a systematic review. Ann Intern Med 166, 257-267.

31. Rippin HL, Hutchinson J, Evans CEL, et al. (2018) National nutrition surveys in Europe: a review on the current status in the 53 countries of the WHO European region. Food Nutr Res 62, 10.2919

32. Newens KJ \& Walton J (2016) A review of sugar consumption from nationally representative dietary surveys across the world. J Hum Nutr Diet 29, 225-240.
33. Huybrechts I, Aglago EK, Mullee A, et al. (2017) Global comparison of national individual food consumption surveys as a basis for health research and integration in national health surveillance programmes. Proc Nutr Soc $76,549-567$.

34. Song SJ \& Song WO (2014) National nutrition surveys in Asian countries: surveillance and monitoring efforts to improve global health. Asia Pac J Clin Nutr 23, 514-523.

35. Australian Bureau of Statistics (2016) Australian Health Survey: Consumption of Added Sugars Australia. Australian Bureau of Statistics [Internet]. Available from: http://www.ausstats.abs.gov.au/ausstats/subscriber.nsf/ 0/58042B7FE6A65821CA2581F4000D1020/\$File/4364.0. 55.011 - australian health survey - consumption of added sugars.pdf.

36. Australian Bureau of Statistics (2014) Australian health survey: nutrition first results - food and nutrients, 2011-12 [Internet]. 4364.0.55.007, 1-78. Available from: http://www.abs.gov. au/AUSSTATS/abs@.nsf/DetailsPage/4364.0.55.0072011-12? OpenDocument.

37. Ridder K, Bel S, Brocatus L, Cuypers K, Lebacq T, Moyersoen I, Ost C, \& Teppers E Enquête de Consommation Alimentaire 2014-2015 Rapport 4: La Consommation Alimentaire. [Internet]. 2016. Available from: https://fcs.wiv-isp.be/nl/ Gedeelde-documenten/FRANS/Rapport4/Resume_rapport_ 4_finaal_finaal.pdf.

38. Ministry of Health Chile (2021) Chile National Food Consumption Survey 2010-2011. Santiago, Chile: Ministry of Health Chile.

39. Trolle E, Gondolf UH, Ege M, Kørup K, Ygil KH \& Christensen T (2013) Danskernes kostvaner Spæd- og småbørn 2006-2007 [Internet]. Available from: http://orbit.dtu.dk/files/84054319/ Rapport_Danskernes_kostvaner_sp_d_og_sm_b_rn_rev_12_ 12_13_1_.pdf.

40. Pitsi T, Zilmer M, Vaask S, et al. (2015) Eesti toitumis- ja liikumissoovitused [Internet]. Available from: https://intra.tai.ee/ images/prints/documents/149019033869_eesti toitumis- ja liikumissoovitused.pdf.

41. Kyttälä $\mathrm{P}$, Ovaskainen M, Kronberg-Kippilä C, et al. (2008) The diet of Finnish preschoolers (in Finnish, abstract in English) [Internet]. Available from: http://www.julkari.fi/ bitstream/handle/10024/78163/2008b32.pdf.

42. Agence nationale de sécurité sanitaire de l'alimentation de, travail l'environnement et du (2017) Étude Individuelle Nationale des Consommations Alimentaires 3 (INCA3) (20142015) Rapport [Internet]. Available from: https://www.anses. $\mathrm{fr} / \mathrm{fr} /$ system/files/NUT2014SA0234Ra.pdf.

43. Pórsdóttir I, Pórisdóttir ÁV \& Pálsson GI (2008) The diet of Icelandic infants: results from a research on infants' diet, growth and iron status 2005-2007 [Internet]. Available from: http://www.landlaeknir.is/servlet/file/store93/item11592/ Ungbarnaskyrsla.pdf.

44. Gunnarsdóttir I, Eysteinsdóttir T, Pórsdóttir T (2008) Hvað borða íslensk börn á leikskólaaldri? Könnun _a mataræði 3ja og 5 á ra barna [Internet]. Available from: http://www. landlaeknir.is/servlet/file/store93/item14897/3ja_og_5_ara_ skyrsla_181208.pdf.

45. Irish Universities Nutrition Alliance (2012) National PreSchool Nutrition Survey 2010-11 [Internet]. Available from: https://irp-cdn.multiscreensite.com/46a7ad27/files/ uploaded/The National Pre-School Nutrition Survey Summary Report June 2012.pdf.

46. Israel Ministry of Health (2017) MABAT Kids - The National Survey on the Health and Nutrition Status of Children Ages 2-11 in Israel: 2015-2016, 79. Available from: https://www. health.gov.il/publicationsfiles/mabat_kids2_11_2015-2016.pdf. 
47. Sette S, Le Donne C, Piccinelli R, et al. (2011) The third Italian National Food Consumption Survey, INRAN-SCAI 2005-06 Part 1: nutrient intakes in Italy. Nutr Metab Cardiovasc Dis 21, 922-932.

48. Sánchez-Pimienta TG, Batis C, Lutter CK, et al. (2016) Sugar-sweetened beverages are the main sources of added sugar intake in the Mexican population. J Nutr 146, 1888S1896S.

49. National Institute for Public Health and the Environment Ministry of Health Welfare and Sport (2019) Food consumption survey 2012-2016 [Internet]. Available from: https:// www.rivm.nl/bibliotheek/rapporten/2020-0083.pdf.

50. Helsedirektoratet (2009) Spedkost - 12 måneder 2006-2007 [Internet]. Available from: https://www.helsedirektoratet.no/ rapporter/spedkost-og-smabarnskost-landsomfattendekostholdsundersokelser/Spedkost 12 måneder - landsomfattende kostholdundersøkelse 2007-2008.pdf/_attachment/ inline/1544ff4a-db55-4db1-b24b-f3efa19dd26b:20d97036e4 aa $5 \mathrm{f} 5 \mathrm{c} 92$.

51. Helsedirektoratet (2009) Småbarnskost - 2 år Landsomfattende kostholdsundersøkelse blant 2 år gamle barn. Available from: åhttps://www.helsedirektoratet.no/rapporter/spedkost-ogsmabarnskost-landsomfattende-kostholdsundersokelser/ Småbarnskost 2007 - landsomfattende kostholdsunder søkelse blant 2 år gamle barn.pdf/_/attachment/inline/ 54fa23d7-f5cc-4ce8-bc21-41418c21a126:5dee89c.

52. Public Health England, Food Standards Agency (2018) National Diet and Nutrition Survey: results from Years 1-4 (combined) of the Rolling Programme [Internet]. Available from: https://assets.publishing.service.gov.uk/government/ uploads/system/uploads/attachment_data/file/699241/NDNS_ results_years_7_and_8.pdf.

53. Bailey RL, Fulgoni VL, Cowan AE, et al. (2018) Sources of added sugars in young children, adolescents, and adults with low and high intakes of added sugars. Nutrients $\mathbf{1 0}$, 102.

54. Herrick KA, Fryar CD, Hamner HC, et al. (2020) Added sugars intake among US infants and toddlers. J Acad Nutr Diet 120, 23-32.

55. US Department of Agriculture (2020) Nutrient intakes from food: mean amounts consumed per individual, by gender and age, what we eat in America, NHANES 2017-2018. Available from: www.ars.usda.gov/nea/bhnrc/fsrg.

56. Elmfada I (2012) Österreichischer Ernährungsbericht 2012 [Internet]. Available from: https://ernaehrungsbericht.univie. ac.at/fileadmin/user_upload/dep_ernaehrung/forschung/ ernaehrungsberichte/oesterr_ernaehrungsbericht_2012.pdf.

57. Souza RAG, Yokoo EM, Sichieri R, et al. (2015) Energy and macronutrient intakes in Brazil: results of the first nationwide individual dietary survey. Public Health Nutr 18, 3086-3095.

58. Statistics Canada (2018) Canadian community health survey - nutrition. Ottawa: Health Canada.

59. Afeiche MC, Koyratty BNS, Wang D, et al. (2018) Intakes and sources of total and added sugars among 4 to 13-year-old children in China, Mexico and the United States. Pediatr Obes 13, 204-212.

60. Pedersen AN, Christensen T, Matthiessen J, Knudsen VK, Sørensen MR, Biltoft-Jensen AP, Fagt S (2015) Danskernes kostvaner 2011-2013 [Internet]. Available from: http://www.food. dtu.dk/-/media/Institutter/Foedevareinstituttet/Publikationer/ Pub-2015/Rapport_Danskernes-Kostvaner-2011-2013.ashx? $\mathrm{la}=\mathrm{da}$.

61. Mensink GBM, Helmut H, Stahl A, Richer A \& Vohmann C (2007) Die aktuelle Nährstoffzufuhr von Kindern und Jugendlichen in Deutschland - Ergebnisse aus EsKiMo,
Ernährungs Umschau [Internet]. Available from: https:// www.ernaehrungs-umschau.de/fileadmin/Ernaehrungs-Ums chau/pdfs/pdf_2007/11_07/EU11_636_646.qxd.pdf.

62. Gunnarsdottir I, Helgadottir H, Thorisdottir B, et al. (2013) Diet of six-year-old Icelandic children - National dietary survey 2011-2012. Laeknabladid Laeknafelag Islands; 99, 17-23.

63. Unit for Nutrition Research (2006) Iceland dietary survey of 9 and 15 year old children and adolescents 2003-2004 [Internet]. Available from: http://www.landlaeknir.is/servlet/file/store93/ item11594/hvad_borda_isl_born_og_ungl.pdf.

64. International Life Sciences Institute India (2019) Consumption levels of sugar among rural and urban population in India [Internet]. Available from: http://www.ilsi-india.org/ (Accessed December 2020).

65. Irish Universities Nutrition Alliance (2019) National Children's Food Consumption Survey II (NCFS II) 2017-2018 [Internet]. Available from: rp-cdn.multiscreensite.com/46a7ad27/files/ uploaded/The National Children $\% 27 \mathrm{~s}$ Food Survey II Summary Report - September 2019.pdf.

66. National Center for Disease Control Israel National Survey on the Subject of Health and Nutrition Status in children aged 2-11 in Israel [Internet]. Available from: https://www.health. gov.il/publicationsfiles/mabat_kids2_11_2015-2016.pdf.

67. Ministry of Health (2003) 2002 National Children's Nutrition Survey (CNS02) [Internet]. Wellington. Available from: https:// www.health.govt.nz/system/files/documents/publications/ nzfoodnzchildren.pdf.

68. FHI (2017) UNGKOST 3 Landsomfattende kosthold sundersøkelse blant 4-åringer i Norge, 2016 [Internet]. Available from: https://www.fhi.no/globalassets/dokumenter filer/rapporter/2016/rapport-ungkost-3-landsomfattendekostholdsundersokelse-blant-4-aringer-i-norge-2016.pdf.

69. FHI (2016) UNGKOST 3 Landsomfattende kostholdsunder søkelse blant elever i 4. -og 8. klasse i Norge, 2015 [Internet]. Available from: https://www.fhi.no/globalassets/ dokumenterfiler/rapporter/2016/ungkost-rapport-24.06.16.pdf.

70. IAN-AF (2017) National Food and Physical Activity Survey (IAN-AF) 2015-2016. ANEXO 2 - INGESTÃO NUTRICIONAL [Internet]. Available from: https://ian-af.up.pt/en/consultade-dados?shs_term_node_tid_depth=All\&field_indicador_ tid=All\&field_estratificacao_tid=All\%0A (accessed December 2020).

71. FEN (2014) Ingesta, perfil y fuentes de energía en la población española: Resultados obtenidos del estudio científico ANIBES. Available from: http://www.fen.org.es/anibes/archivos/ documentos/ANIBES_numero_3.pdf.

72. Ruiz E, Rodriguez P, Valero T, et al. (2017) Dietary intake of individual (free and intrinsic) sugars and food sources in the Spanish population: findings from the ANIBES study. Nutrients 9, 275.

73. Roberts C, Steer T, Maplethorpe N, et al. (2018) National diet and nutrition survey: results from Years 7 and 8 (combined) of the Rolling Programme (2014/2015-2015/2016). London: Public Health England.

74. d'Andorra G, de Salut M \& i Família BS (2007) Evaluation of the nutritional status of the Andorran population [Internet]. Available from: http://www.salut.ad/images/microsites/ AvaluacioNutricional_04-05/index.html (accessed December 2020).

75. Langlois K, Garriguet D, Gonzalez A, et al. (2019) Change in total sugars consumption among Canadian children and adults. Health Rep 30, 10-19.

76. Perrar I, Schadow AM, Schmitting S, et al. (2020) Time and age trends in free sugar intake from food groups among children and adolescents between 1985 and 2016 . Nutrients 12, 20. 
77. Irish Universities Nutrition Alliance (IUNA) (2007) National Teens' Food Survey 2005-2006. [Internet]. Available from: https://irp-cdn.multiscreensite.com/46a7ad27/files/uploaded/ The National Teens\%27 Food Survey \%282005-2006\%29.pdf.

78. Joyce T, Mccarthy SN \& Gibney MJ (2008) Relationship between energy from added sugars and frequency of added sugars intake in Irish children, teenagers and adults. $\mathrm{Br} J$ Nutr 99, 1117-1126.

79. Israel Center for Disease Control (2017) Mabat Youth Second National Health and Nutrition Survey of 7 th-12th grade students 2015-2016. Publication 373 [Internet]. Available from: https://health.gov.il/publicationsfiles/mabat_kids2_11_20152016-eng.pdf\%0A\%0A.

80. Kibblewhite R, Nettleton A, McLean R, et al. (2017) Estimating free and added sugar intakes in New Zealand. Nutrients $\mathbf{9}$, 1292.

81. University of Otago and Ministry of Health (2011) A focus on nutrition: key findings of the 2008/09 New Zealand Adult Nutrition Survey. Wellington: Ministry of Health.

82. Mis NF, Kobe H, Štimec M. (2012) Dietary intake of macro- and micronutrients in Slovenian adolescents: comparison with reference values. Ann Nutr Metab 61, 305-313.

83. Rust P, Hasenegger V \& König J (2017) Österreichischer Ernährungsbericht [Internet]. Available from: https:// broschuerenservice.sozialministerium.at/Home/Download? publicationId $=528 \% 0 \mathrm{~A} \% 0 \mathrm{~A}$ (accessed December 2020).

84. Valsta L, Kaartinen N, Tapanainen H, Männistö S \& Sääksjärvi K (2018) Ravitsemus Suomessa - FinRavinto 2017 -tutkimus. Terveyden ja hyvinvoinnin laitos (THL). Raportti 12/2018, 239 sivua [Internet]. Available from: https://www.julkari.fi/ bitstream/handle/10024/137433/URN_ISBN_978-952-343238-3.pdf?sequence=1\&isAllowed $=\mathrm{y} \% 0 \mathrm{~A} \% 0 \mathrm{~A} \% 0 \mathrm{~A} \quad($ accessed December 2020).

85. Max Rubner-Institut (2008) Nationale Verzehrsstudie II Ergebnisbericht Teil 1; Max Rubner-Institut Karlsruhe: Karlsruhe [Internet]. Available from: https://www.bmel.de/ SharedDocs/Downloads/Ernaehrung/NVS_Ergebnisbericht Teil2.pdf?_blob=publicationFile\%0A\%0A （accessed December 2020).

86. Cust AE, Skilton MR, van Bakel MME, et al. (2009) Total dietary carbohydrate, sugar, starch and fibre intakes in the European prospective investigation into cancer and nutrition. Eur J Clin Nutr 63, S37-S60.

87. Eszter SN, Márta B, Éva I, et al. (2017) Országos táplálkozás és tápláltsági állapot vizsgálat - OTÁP2014. II. A magyar lakosság energia-és makrotápanyag-bevitele. Orv Hetil 158, 587-597.

88. Directorate of Health (2011) Hvað borða Íslendingar? Könnun á mataræði Íslendinga 2010-2011 Helstu niðurstöður 20102011. Helstu niðurstöður [Internet]. Available from: ðhttp:// www.landlaeknir.is/servlet/file/store93/item14901/Hvað borða Íslendingar_april 2012.pdf.

89. Irish Universities Nutrition Alliance (IUNA) (2011) National adult nutrition survey: food and nutrient intakes, physical measurements, physical activity patterns and food choice motives. Summary Report. [Internet]. Available from: https:// irp-cdn.multiscreensite.com/46a7ad27/files/uploaded/The\%20 National\%20Adult\%20Nutri.

90. Walton J, Kehoe L, McNulty BA, et al. (2017) Intakes and sources of dietary sugars in a representative sample of Irish adults (18-90y). Proc Nutr Soc [Internet]. Cambridge University Press; [cited 2021 Jan 4]; 76, E65. Available from: https://www. cambridge.org/core/product/identifier/S0029665117001380/ type/journal_article.

91. Israel Center for Disease Control (2019) Rav Mabat Adult Second National Health and Nutrition Survey 2014-2016.
Publication 383 [Internet]. Available from: https://health.gov. il/publicationsfiles/mabat_adults_2014_2016_383_en.pdf.

92. Barzda A, Bartkeviciute R, Baltusyte I, Stukas R \& Bartkeviciute S (2016) Suaugusiu ir pagyvenusiu c Lietuvos gyventoju ${ }_{c}$ faktin 'es mitybos ir mitybos $1_{c}$ pro ${ }^{`}{ }^{2} u_{c}$ tyrimas. Visuom. Sveik 72, 85-94. [Internet]. Available from: http:// www.hi.lt/uploads/pdf/visuomenes sveikata/2016.01.72/VS 2016 1(72) ORIG Mitybos iprociai.pdf \%0A\%0A.

93. Helsedirektoratet (2012) Norkost 3 En landsomfattende kostholdsundersøkelse blant menn og kvinner i Norge I alderen 18-70ar, 2010-11 [Internet]. Available from: https:// helsedirektoratet.no/Lists/Publikasjoner/Attachments/301/ Norkost-3-en-landsomfattende-kostholdsundersokelse-blantmenn-og-kvinner-i-norge-i-alderen-18-70-ar-2010-11-IS-2000. pdf.

94. Chatelan A, Gaillard P, Kruseman M, et al. (2019) Total, added, and free sugar consumption and adherence to guidelines in Switzerland: results from the First National Nutrition Survey menuCH. Nutrients [Internet]. MDPI AG; [cited 2020 Jun 10]; 11. Available from: http://www.ncbi.nlm.nih.gov/pubmed/ 31109151.

95. Heuer T (2017) Estimation of "free" sugar intake in Germany based on the data of the German National Nutrition Survey II. Available from: https://www.openagrar.de/receive/openagrar_ mods_00030594.

96. De la Cruz-Góngora V, Martínez-Tapia B, Cuevas-Nasu L, et al. (2017) Dietary intake and adequacy of energy and nutrients in Mexican older adults: Results from two National Health and Nutrition Surveys. Salud Publica Mex 59, 285-298.

97. Azaïs-Braesco V, Sluik D, Maillot M, et al. (2017) A review of total \& added sugar intakes and dietary sources in Europe. Nutr J BioMed Central Ltd. 16, 6.

98. IUNA (2019) National Children's Food Survey II: summary report [Internet], 1-44. Available from: www.iuna.net.

99. Hayes M (2019) How many countries are there in the world? [Internet]. WorldAtlas, [cited 2020 Jun 15]. Available from: https://www.worldatlas.com/articles/how-many-countriesare-in-the-world.html.

100. Micha R, Coates J, Leclercq C, et al. (2018) Global dietary surveillance: data gaps and challenges. Food Nutr Bull 39, 175-205.

101. Louie JCY, Moshtaghian H, Boylan S, et al. (2015) A systematic methodology to estimate added sugar content of foods. Eur J Clin Nutr 69, 154-161.

102. Swan GE, Powell NA, Knowles BL, et al. (2018) A definition of free sugars for the UK. Public Health Nutr 21, 1636-1638.

103. Amoutzopoulos B, Page P, Steer T, et al. (2020) Free and added sugar consumption and adherence to guidelines: the UK national diet and nutrition survey (2014/15-2015/16). Nutrients MDPI AG, 12, 393.

104. Amoutzopoulos B, Steer T, Roberts C, et al. (2018) A disaggregation methodology to estimate intake of added sugars and free sugars: an illustration from the UK national diet and nutrition survey. Nutrients MDPI AG; 10,1177.

105. Davy B \& Jahren H (2016) New markers of dietary added sugar intake. Curr Opin Clin Nutr Metab Care 19, 282-288.

106. Abreu TC, Hulshof PJM, Boshuizen HC, et al. (2020) Validity coefficient of repeated measurements of urinary marker of sugar intake is comparable to urinary nitrogen as marker of protein intake in free-living subjects. Cancer Epidemiol Biomarkers Prev 30, 193-202.

107. Mela DJ (2020) A proposed simple method for objectively quantifying free sugars in foods and beverages. Eur J Clin Nutr [Internet] 74, 1366-1368. doi: 10.1038/s41430-0200575-x 
108. Louie JCY (2020) Objective biomarkers for total added sugar intake - are we on a wild goose chase? Adv Nutr 11, 1429-1436.

109. Capacci S, Allais O, Bonnet C, et al. (2019) The impact of the French soda tax on prices and purchases. An ex post evaluation. PLoS One 14, e0223196.

110. World Health Organisation (2017) Reformulation measures for foods and beverages - European Health Information Gateway [Internet], [cited 2020 Jun 15]. Available from: https://gateway.euro.who.int/en/indicators/gnpr_survey_ 17-reformulation-measures-for-foods-and-beverages/.

111. Public Health England (2018) Sugar reduction and wider reformulation programme: report on progress towards the first $5 \%$ reduction and next steps May 2018 About Public Heath England [Internet]. Available from: www.facebook.com/ PublicHealthEngland.

112. Wright A, Smith KE \& Hellowell M (2017) Policy lessons from health taxes: a systematic review of empirical studies. $B M C$ Public Health 17, 583.

113. Redondo M, Hernández-Aguado I \& Lumbreras B (2018) The impact of the tax on sweetened beverages: a systematic review. Am J Clin Nutr 108, 548-563.

114. Briggs ADM, Mytton OT, Kehlbacher A, et al. (2013) Overall and income specific effect on prevalence of overweight and obesity of $20 \%$ sugar sweetened drink tax in UK: econometric and comparative risk assessment modelling study. BMJ 347, f6189.
115. Blakely T, Nghiem N, Genc M, et al. (2020) Modelling the health impact of food taxes and subsidies with price elasticities: the case for additional scaling of food consumption using the total food expenditure elasticity. PLoS One 15, e0230506

116. Ma Y, He FJ, Yin Y, et al. (2016) Gradual reduction of sugar in soft drinks without substitution as a strategy to reduce overweight, obesity, and type 2 diabetes: a modelling study. Lancet Diabetes Endocrinol 4, 105-114.

117. Amies-Cull B, Briggs ADM \& Scarborough P (2019) Estimating the potential impact of the UK government's sugar reduction programme on child and adult health: modelling study. BMJ 365.

118. Center for Food Safety and Applied Nutrition (2018) Labeling \& nutrition - changes to the nutrition facts label. Washington DC: United States Department of Health \& Human Services.

119. U.S. Department of Agriculture \& Global Agricultural Information Network (2020) Implementation of the New Mexican Regulation for front-of-package nutrition label.

120. Tierney M, Gallagher AM, Giotis ES, et al. (2017) An online survey on consumer knowledge and understanding of added sugars. Nutrients $\mathbf{9}, 37$.

121. Tang Q, Lin Q, Yang Q, et al. (2020) Knowledge, attitude, and practice of adolescent parents on free sugar and influencing factors about recognition. Int J Environ Res Public Health 17, 4003 . 